\title{
The Use of Social Media to Increase the Impact of Health Research: Systematic Review
}

Marco Bardus ${ }^{1}$, BA, MA, PhD; Rola El Rassi², MSc; Mohamad Chahrour ${ }^{3}$, BSc; Elie W Akl ${ }^{3}$, MD; Abdul Sattar Raslan $^{3}$, BSc; Lokman I Meho ${ }^{4}$, BA, MA, MSLS, PhD; Elie A Akl ${ }^{5}, \mathrm{MD}, \mathrm{MPH}, \mathrm{PhD}$

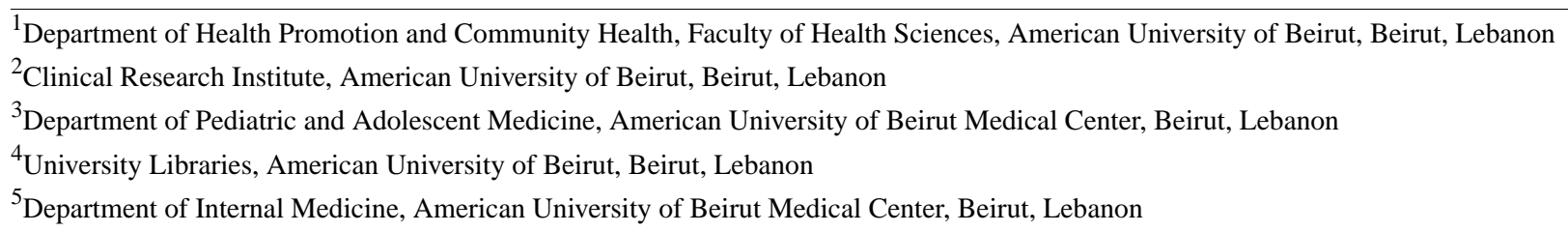

Corresponding Author:

Elie A Akl, MD, MPH, PhD

Department of Internal Medicine

American University of Beirut Medical Center

PO Box 11-0236, Riad-El-Solh

Beirut, 11072020

Lebanon

Phone: 9611374374

Email: ea32@aub.edu.lb

\section{Abstract}

Background: Academics in all disciplines increasingly use social media to share their publications on the internet, reaching out to different audiences. In the last few years, specific indicators of social media impact have been developed (eg, Altmetrics), to complement traditional bibliometric indicators (eg, citation count and h-index). In health research, it is unclear whether social media impact also translates into research impact.

Objective: The primary aim of this study was to systematically review the literature on the impact of using social media on the dissemination of health research. The secondary aim was to assess the correlation between Altmetrics and traditional citation-based metrics.

Methods: We conducted a systematic review to identify studies that evaluated the use of social media to disseminate research published in health-related journals. We specifically looked at studies that described experimental or correlational studies linking the use of social media with outcomes related to bibliometrics. We searched the Medical Literature Analysis and Retrieval System Online (MEDLINE), Excerpta Medica dataBASE (EMBASE), and Cumulative Index to Nursing and Allied Health Literature (CINAHL) databases using a predefined search strategy (International Prospective Register of Systematic Reviews: CRD42017057709). We conducted independent and duplicate study selection and data extraction. Given the heterogeneity of the included studies, we summarized the findings through a narrative synthesis.

Results: Of a total of 18,624 retrieved citations, we included 51 studies: 7 (14\%) impact studies (answering the primary aim) and $44(86 \%)$ correlational studies (answering the secondary aim). Impact studies reported mixed results with several limitations, including the use of interventions of inappropriately low intensity and short duration. The majority of correlational studies suggested a positive association between traditional bibliometrics and social media metrics (eg, number of mentions) in health research.

Conclusions: We have identified suggestive yet inconclusive evidence on the impact of using social media to increase the number of citations in health research. Further studies with better design are needed to assess the causal link between social media impact and bibliometrics.

(J Med Internet Res 2020;22(7):e15607) doi: 10.2196/15607

\section{KEYWORDS}

social media; research; bibliometrics; Altmetrics; journal impact factor; translational medical research 


\section{Introduction}

\section{Social Media and Its Impact in Health Research}

Individual researchers and academic institutions use social media to disseminate their research findings to a broad audience that includes the public and health care practitioners. According to Carr and Hayes [1], social media can be broadly defined as "internet-based, disentrained, and persistent channels of mass personal communication facilitating perceptions of interactions among users, deriving value primarily from user-generated content". For example, social media can be used by clinicians who would benefit from the findings in dealing with patients. A recent scoping review of the literature on social media use in health research revealed that social media are increasingly used to recruit patients, collect data, and establish and maintain user engagement, especially in the name of research dissemination [2]. At the same time, in the era of the internet and web 2.0 technologies that allow content generation and sharing [3], social media have been increasingly used as a source for measuring the impact of research, as they contribute to knowledge generation, dissemination, and translation [4] Indeed, social media are increasingly required to measure their research performance and demonstrate the value of their research to governments and funding organizations [5]. Researchers in the domain of health and medical sciences have been particularly concerned about demonstrating the impact of their work as it usually bears implications for public health [6]. Hence, measuring the impact of health research is essential for influencing policy-making processes, improving health systems, and health-related socioeconomic impact [6]. According to the research impact framework (RIF) for health research [4], the domain of research-related impact is generally and traditionally evaluated according to conventional and traditional bibliometric approaches, which generally include the number of citations, the impact factor (IF), or the h-index [7]. Some academic institutions in the United States, Canada, and Europe started including social media impact as an evaluation criterion in their tenure and promotion policies [8]. According to the systematic review by Cruz Rivera et al [6] on the impact of health care research, researchers should consider indicators such as "the number of reads for published articles; article download rate and number of journal webpage visits; and citations rates in non-journal media such as newspapers and mass and social media (ie, Twitter and blogs)".

Researchers can now discuss their papers and share their publications on various social networking sites [9] or with the general public interested in their topic. Using typology of social media by Constantinides and Fountain [10], health researchers can use 5 types of dissemination platforms: (1) blogs or web-based journals; (2) social networking sites, such as Facebook or Twitter, or professionally oriented platforms such as LinkedIn, ResearchGate, and Academia, which have recently emerged as social networking sites for academics [11]; (3) content communities; (4) forums or bulletin boards; and (5) content aggregators, such as Diigo, CiteULike, Delicious, Evernote, or through reference management software such as EndNote, Mendeley, RefWorks, Papers, and Zotero. These software companies have developed proprietary communities of users who share citations. In this way, an article can reach new and widespread audiences, broader than those of the limited subscribers of academic journals whose content is generally protected by paywalls. Some publishers have also embraced the movement of social media dissemination by including options for authors to semiautomatically share their output on academic content aggregators that include dashboards to measure the reach and impact of social media posts, based on the link or digital object identifier (DOI) associated with each article. Examples include free platforms such as the nonprofit-owned ImpactStory; or the profit-oriented Kudos, independent from publishing houses; or Publons, owned and managed by Clarivate Analytics; and PlumX [12], owned by Elsevier.

\section{Social Media Impact Measures}

How is social media impact defined and measured? In the late 1990s, researchers started studying the phenomenon of internet-based dissemination of knowledge and information, forging the terms of webometrics or cybermetrics [13]. In 2010, a group of researchers defined a new set of metrics, Altmetrics (which stands for alternative metrics) that include web-based metrics (eg, number of link shares, likes, tweets, and views) and qualitative data that are complementary to traditional, citation-based metrics [14]. The so-called Altmetrics attention score (AAS) includes various indices of performance of a paper, such as the number of views; the number of discussions on social media (tweets, Facebook posts, and Wikipedia pages); recommendations (eg, Faculty of 1000); saved articles on popular social bookmarking services such as Mendeley or CiteULike; and the number of citations obtained from Google Scholar, CrossRef, PubMed Central, and Scopus [15]. Despite some intrinsic limitations - as the AAS can be inflated by self-citations, automatic retweeting, or sharing on various social media platforms [7]-Altmetrics is considered the current standard for measuring the impact of research on the internet and beyond [14-16].

Similar to the number of citations, the number of social media mentions of an article is a function of time since its publication. In 2011, Eysenbach published, in this journal, a seminal paper entitled, Can tweets predict citation metrics on social media [17]. The author proposed a set of measures for social media impact that would account for the dimension of time. The paper introduced the concepts of Tweetations, Twimpactfactor, and Twindex. Tweetations were defined as "citations in a tweet," that is, tweets mentioning the exact journal article URL (hence excluding links to DOI, PubMed entries, or other links). The twimpact factor was defined as the "cumulative number of tweetations within n days after publication" (eg, tw7 means the total number of tweetations after 7 days) [17]. The Twindex (or tweetation index) was defined as a "metric ranging from 0 to 100 indicating the relative standing of an article compared to other articles." It is based on a percentile, rank-ordering of an article by tweetations, relative to other articles published in the same journal, which were published around the same time [17]. The author recommended that papers investigating the relationship between citations and social media mentions should adjust for time since the publication of an article (or specify a timeframe when these metrics were obtained), journal type, 
seasonal variations, and other possible confounders that might explain a nonlinear distribution of social media mentions.

\section{Social Media and Bibliometrics}

How is social media dissemination related to citations? Social media dissemination is generally associated with the higher reach of an article. Some research, sponsored by Academia.edu, showed that by sharing an article on this specific academic, social networking site, a researcher could receive up to $69 \%$ more citations over 5 years [18]. In Eysenbach's (2011) paper, the author found a positive correlation between social media and subsequent citations and social media [17]. However, to what extent is there a causal link between the use of social media and subsequent citations? In other words, what is the impact of social media on citations and bibliometric indicators? To the best of our knowledge, to date, there is only one experimental study, using a randomized controlled trial (RCT) design, that has clearly shown a causal relationship between subsequent citations and the dissemination of articles through a proprietary web-based distribution platform (TrendMD) [19]. Although TrendMD does not meet the definition of social media as intended in this paper [1], as it is not based on user-generated content, but on content that is pushed by an algorithm of sponsored recommendations, the study provides a useful benchmark. The authors found significant effects on citations and Mendeley saves for the intervention group (TrendMD) compared with the control (no diffusion on TrendMD) after 6 and 12 months [19]. In addition, the intervention had positive effects on citations at both 6 and 12 months for articles in the area of health and medical sciences [19].

However, evidence from systematic reviews on this matter seems to be scarce. A basic search for systematic reviews on Altmetrics on Google Scholar, on April 2, 2019 (in titles of articles) yielded only 24 results, 3 of which were reviews: 1 systematic review mapping the evidence for marketing research [20] and 2 literature reviews on Altmetrics used for generic scholarly research output [21,22]. In terms of health research, the review evidence is also limited. To the best of our knowledge, there is only one ongoing systematic review-whose protocol is registered in the International Prospective Register of Systematic Reviews (PROSPERO) [23] - that is aimed at evaluating the use of social media to disseminate research findings among health care professionals. A similar research conducted on April 2, 2019, on PubMed, yielded 18 hits, 2 of which were systematic reviews: the first focused on medical research output and reported significant associations between Altmetrics and traditional citations, without linking measures of impact [24]; the second is a systematic review of reviews and meta-analyses and focused on the evaluation of methodological quality in the domain of skin psoriasis [25]. The latter reported that a journal's IF could predict the number of tweets, whereas the years of publication and number of Mendeley readers predicted the number of citations on Google Scholar. Nevertheless, the authors concluded there does not seem to be a connection between scientific quality, social media, activity, and article usage [25].

Therefore, the primary aim of this study was to systematically review the literature on the impact of using social media on the dissemination of health research. The secondary aim was to assess the correlation between Altmetrics and traditional citation-based metrics.

\section{Methods}

\section{Protocol and Eligibility Criteria}

We developed a priori protocol for this systematic review and registered it in the PROSPERO database (CRD42017057709) [23]. This review focuses on health research, which includes biomedical, epidemiological, clinical, public health, and health systems research [26]. The inclusion criteria were as follows:

- Population: The unit of analysis of this review is studies (ie, study reports) published in health-related journals, including primary and secondary research, and editorials.

- Interventions/exposures: For both aims, we included studies that evaluated the use of social media to disseminate health research, which reported the outcomes described below. In the protocol, we included examples of social media and web 2.0 applications, defined as "a group of internet-based applications that build on the ideological and technological foundations of web 2.0, and that allow the creation and exchange of user generated content" [27]. Web 2.0 applications included interactive websites, blogging platforms, social networking, and social sharing sites, as described in the Introduction section.

- Comparator: The comparator for the primary aim (assessing the impact) was not using social media.

- Outcome measures: To be included, studies had to report measures of research dissemination such as traditional bibliometrics, Altmetrics, or webometrics indicators. Traditional bibliometric indicators were defined as quantitative data and statistics to publications such as journal articles and their accompanying citation counts. Altmetrics were defined as web-sourced metrics and qualitative data that are complementary to traditional, citation-based bibliometrics [15]. Webometrics were defined as the study of the quantitative aspects of the construction and use of information resources, structures, and technologies on the web, by drawing on bibliometric and infometric approaches [13]. Studies were included if they measured Altmetrics about webometrics and traditional bibliometrics, without any restrictions to any specific types of metrics.

- Study design: Experimental studies (eg, RCTs and nonrandomized studies or cohort studies), case series, and case studies.

- Publication type: We included original research papers, including scientific meeting abstracts or research letters, if they contained sufficient information to fill the extraction forms.

\section{Search Strategy}

We searched the following electronic databases on July 12, 2017: Medical Literature Analysis and Retrieval System Online (MEDLINE; access via Ovid), Excerpta Medica dataBASE (EMBASE; access via Ovid), and Cumulative Index to Nursing and Allied Health Literature (CINAHL; access via EBSCO). We updated the searches on August 22, 2019. We developed a 
search strategy with the help of a health information specialist with experience in systematic reviews. The search strategy encompassed 2 main concepts: social media (based on the search strategy of a related systematic review by one of the authors [28]) and impact on dissemination (including bibliometrics, Altmetric and academic impact). The search strategy used both keywords and medical subject heading terms. The search strategies used are provided in Multimedia Appendix 1.

We did not use language restrictions. We did restrict the search timeframe by using the start date of January 2005, the year of the introduction of the web 2.0 concept [3]. We also reviewed the reference lists of relevant papers and searched our files for both published and unpublished studies.

\section{Selection Process}

Before starting the selection process, we conducted calibration exercises to train the reviewers and clarify the eligibility criteria. In all, 3 reviewers (EWA, MC, and ASR) and 2 research assistants screened the titles and abstracts of identified references in duplicate and independently for potential eligibility.

We obtained the full texts for all references judged as potentially eligible by at least one reviewer through our local library. For articles not found through the library, we searched on Google, Google Scholar, ResearchGate, or Academia.edu to locate self-archived copies. We contacted corresponding authors via ResearchGate or emailed them to obtain a copy of the articles. Then, the same 3 reviewers screened the full texts in duplicate and independently for eligibility, using a standardized pilot-tested full-text screening form. Once acceptable interrater reliability was achieved, the reviewers completed the selection tasks independently. All disagreements were resolved by discussion and with the help of a third reviewer (MB), who double-checked all decisions and confirmed the reasons for exclusion.

We assessed the interrater reliability for titles and abstract screening using Gwet $\mathrm{AC} 1$ index, as it is less prone to bias when there is a large disproportion in binary categories (ie, excluded vs included articles) that are not judged as relevant [29,30]. We assessed the interrater reliability for full-text screening using the kappa statistic.

\section{Data Abstraction Process}

The review teams abstracted data from eligible studies in duplicate and an independent manner, using a standardized and pilot-tested data abstraction form with detailed instructions. Disagreements were resolved through discussions and with the help of a third reviewer (MB and EAA). Any inconsistencies in the abstraction tables were discussed within the research team (MB, EAA, RER, EWA, MC, and ASR) until consensus was reached. The abstracted data items included:

1. general information about the report, such as the first author's name, year of publication, type of study (eg, experimental, cross-sectional, cohort, or qualitative), health area, journal, population/unit of analysis, sources of data, and period of investigation;
2. metrics reported, such as type of social media used and type of metrics (eg, social media metrics, such as Altmetric attention score; bibliometrics, such as citations; and webometrics, such as page views and number of downloads);

3. results, as reported by the study authors; and

4. funding and reported conflicts of interest.

For experimental studies, we collected additional specific information about the intervention (eg, sample size, frequency and reach of the intervention, duration and frequency of the intervention, and profile owner) and control conditions, where applicable. We also extracted the information about effects (eg, $F$ tests and $t$ tests) and $P$ values, as reported by the authors. We used a web-based effect size calculator [31] to estimate effect sizes if they were not included in the original publication. One author (MB) checked all abstraction tables for consistency.

When correlations between social media metrics and citations were reported, following Eysenbach recommendations [17], we also extracted details about whether the paper: (1) reported social media metrics adjusted for time (eg, Twimpact factor) or provided a rationale for selecting a timeframe to assess the relationship, (2) included social media metrics that adjust for some kind of confounders (eg, using Twindex metric or stratifying by article type and/or topic), (3) the type of correlation test used (eg, using Spearman rho and/or Pearson $r$ ), and (4) explored the correlation using scatterplots or employed tests for nonlinear relationships (eg, log-linear and/or nonparametric tests).

\section{Data Synthesis}

Given the heterogeneity of the included studies in terms of characteristics of the population, health area, study design, and reported outcomes (including $P$ values and correlation coefficients), we summarized the findings through a narrative synthesis. In the summary tables included in this review, we reported $P$ values and correlation coefficients and measures of effect sizes, as explicitly mentioned by the authors of the selected studies. We followed Journal of Medical Internet Research's convention for reporting $P$ values (3 digits) and correlation coefficients ( 2 digits). We included the text of the original source in quotation marks. For studies reporting correlations between social media and citation metrics, we defined the methodological quality of the paper using 4 indicators, using Eysenbach (2011) paper as a benchmark [17]: (1) appropriately adjusting for time, (2) appropriately adjusting for confounders, (3) appropriately exploring correlations, and (4) appropriately reporting nonlinear correlations tests and statistics. Appropriately adjusting for time means that the article accounted for the variability in the metric by time or specified a time when the social media metric was obtained about the time of data analysis. Appropriately adjusting for confounders means that the social media metric was adjusted for confounders, such as journal type, article type, and for the journal and the season, for example by using the Twindex metric, which is a percentile ranking relative to other articles published in the same journal and the same period. Appropriately exploring correlations involves the authors checking for correlations between social media metrics and bibliometrics by inspecting 
scatterplots. Appropriate reporting of tests for nonlinear correlations, such as Spearman rho correlations, log-linear tests of relationships, or other statistics, was based on ranking for non-normal distributions in the citations and social media mentions. We used the following scoring convention: (1) accounting for the time when selecting the data timeframe or acknowledging the role of time since publication $(+)$ and adjusting the social media metric for time $(++) ;(2)$ appropriately adjusting for confounders such as article type, topic, and/or subject (+) and seasonality or time factors using Twindex or similar metrics that account for the relative ranking of the article to the journal and season (++); (3) appropriately exploring correlations by including scatterplots (+); and (4) appropriately reporting nonlinear correlation tests and statistics $(+)$ as well as log-linear relationship tests $(++)$.

\section{Results}

\section{Study Selection}

Figure 1 shows the Preferred Reporting Items for Systematic Reviews and Meta-Analyses (PRISMA) flow diagram. Searches in the selected electronic databases yielded 13,576 records in July 2017 and 5048 in August 2019, totaling 18,624. Of these, 577 were selected for full-text screening, following a duplicate and independent selection process. The level of agreement at the title and abstract screening phase was high (mean Gwet AC1 0.96, SD 0.03). Similarly, we excluded 521 of 577 full-text records, achieving a substantial interrater agreement (mean Kappa 0.69, SD 0.22; mean Gwet AC1 0.77, SD 0.04). We excluded these 521 articles for the following reasons (a list of excluded records is provided in Multimedia Appendix 2): 198 did not discuss health-related research; 116 discussed social media application use, but not for disseminating research; 105 were not original research articles (ie, editorials, commentaries, conceptual papers, literature reviews, praising the use of social media for research dissemination); 54 did not report relevant study outcomes (ie, focusing either on Altmetrics, social media, or citation metrics separately); and 18 were records discussing the use of social media to increase the impact of a journal. Other reasons for exclusion were as follows: 26 were duplicates and 4 were citations of conference abstracts. After consensus-seeking discussions, we judged 56 records as eligible for inclusion in this systematic review, representing 51 unique studies, as 5 studies presented the same data in different publications (an abstract followed by a publication in a journal). The studies were by Amath [32,33], Hayon [34], Knight [35,36], Nolte $[37,38]$, and O'Connor [39,40]. A total of 5 articles included only a conference abstract but were deemed to provide sufficient data for inclusion [41-45]. 
Figure 1. PRISMA flow diagram. MEDLINE=Medical Literature Analysis and Retrieval System Online, EMBASE=Excerpta Medica dataBASE, CINAHL=Cumulative Index to Nursing and Allied Health Literature.

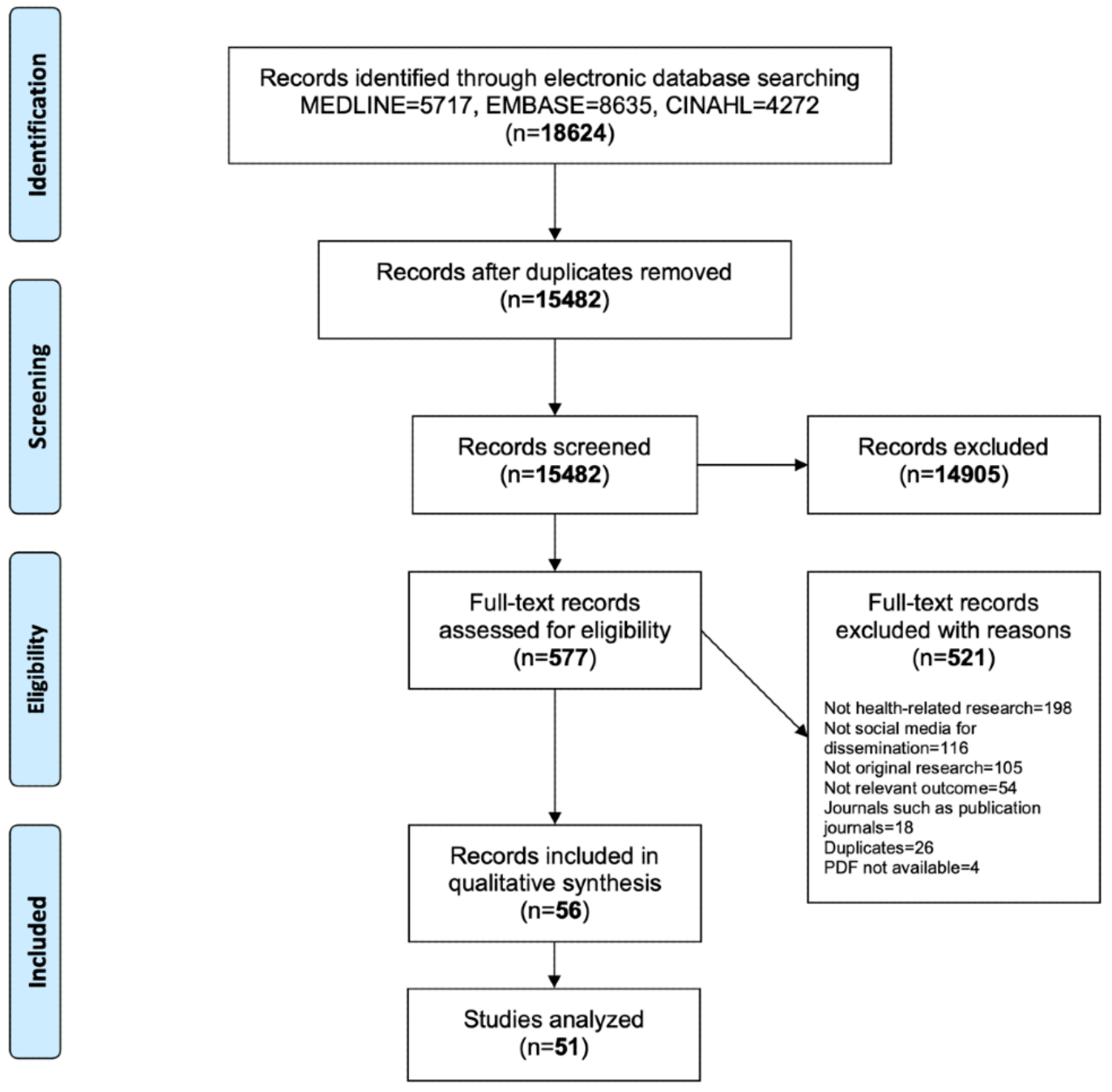

\section{Characteristics of the Included Studies}

A total of 7 of 51 studies (14\%) were categorized as impact studies [46-52], as they presented some interventions that tested the use of social media to disseminate research articles. The main characteristics of impact studies are summarized in Table 1. In all, $86 \%(44 / 51)$ studies were categorized as correlational studies [16,17,25,32,34,36,38,39,41-45,53-83], as they described the associations between traditional bibliometrics and Altmetrics. Correlational studies were classified according to the number of quality indicators as follows: very good quality, with 4/4 indicators (7/44, 16\% studies; Table 2); good quality, with $3 / 4$ indicators $(8 / 44,18 \%$ studies; Table 3$)$; fair quality, with $2 / 4$ indicators $(10 / 44,23 \%$ studies; Table 4$)$; poor quality, with $1 / 4$ indicators (12/44, 27\% studies; Table 5); and very poor quality, with $0 / 4$ indicators (7/44, 16\% studies; Table 6). Detailed information of all 51 studies is included in Multimedia Appendix 3. In the next paragraphs, the results are presented separately, following the primary and secondary aims of this study. 
Table 1. Characteristics of studies assessing the impact of social media interventions ( $\mathrm{n}=7$ ).

\begin{tabular}{|c|c|c|c|c|c|c|}
\hline References & $\begin{array}{l}\text { Health re- } \\
\text { search area }\end{array}$ & $\begin{array}{l}\text { Unit and period of } \\
\text { analysis }\end{array}$ & Type of study & Social media interventions & Metrics reported & Results \\
\hline $\begin{array}{l}\text { Allen, } \\
2013[48]\end{array}$ & $\begin{array}{l}\text { Clinical pain } \\
\text { sciences }\end{array}$ & $\begin{array}{l}16 \text { original research } \\
\text { articles from } P L O S \\
O N E(2006-2011)\end{array}$ & $\begin{array}{l}\text { Quasi-experimen- } \\
\text { tal (before-after) }\end{array}$ & $\begin{array}{l}\text { Blog posts shared on Face- } \\
\text { book, Twitter, LinkedIn, and } \\
\text { ResearchBlogging.org }\end{array}$ & $\begin{array}{l}\text { Citations (Sco- } \\
\text { pus); HTML } \\
\text { views and PDF } \\
\text { downloads }\end{array}$ & $\begin{array}{l}\text { Significant increase in } \\
\text { HTML and PDF views; } \\
\text { no significant effect on } \\
\text { citations approximately } \\
1 \text { year after publication }\end{array}$ \\
\hline $\begin{array}{l}\text { Cawcutt } \\
2019[50]\end{array}$ & $\begin{array}{l}\text { Women's } \\
\text { health }\end{array}$ & $\begin{array}{l}8 \text { original research ar- } \\
\text { ticles published in } 8 \\
\text { journals (2018) }\end{array}$ & $\begin{array}{l}\text { Quasi-experimen- } \\
\text { tal (before-after) }\end{array}$ & $\begin{array}{l}\text { Tweets chatted during a } \\
\text { Physician's Weekly tweet } \\
\text { chat event (\#PWChat) }\end{array}$ & $\begin{array}{l}\text { Article down- } \\
\text { loads; } \text { AAS }^{\text {a }}\end{array}$ & $\begin{array}{l}\text { Increased AAS; in- } \\
\text { creased downloads (statis- } \\
\text { tical significance not re- } \\
\text { ported) }\end{array}$ \\
\hline $\begin{array}{l}\text { Fox, } 2015 \\
{[46]}\end{array}$ & Cardiology & $\begin{array}{l}243 \text { articles, } 121 \text { inter- } \\
\text { vention and } 122 \text { con- } \\
\text { trol, available from } \\
\text { Circulation journal } \\
(2013-2014)\end{array}$ & $\begin{array}{l}\text { Experimental } \\
\left(\mathrm{RCT}^{\mathrm{b}}\right)\end{array}$ & $\begin{array}{l}\text { Twitter and Facebook posts } \\
\text { through the official circula- } \\
\text { tion of social media ac- } \\
\text { counts }\end{array}$ & $\begin{array}{l}\text { HTML views and } \\
\text { PDF downloads }\end{array}$ & $\begin{array}{l}\text { No significant difference } \\
\text { in } 30 \text { days' HTML views } \\
\text { (and downloads) }\end{array}$ \\
\hline $\begin{array}{l}\text { Fox, } 2016 \\
{[47]}\end{array}$ & Cardiology & $\begin{array}{l}152 \text { articles, } 74 \text { inter- } \\
\text { vention and } 78 \text { con- } \\
\text { trol, available from } \\
\text { Circulation journal } \\
\text { (2015) }\end{array}$ & $\begin{array}{l}\text { Experimental } \\
(\mathrm{RCT})\end{array}$ & $\begin{array}{l}\text { Twitter and Facebook posts } \\
\text { through the official circula- } \\
\text { tion of social media ac- } \\
\text { counts }\end{array}$ & $\begin{array}{l}\text { HTML views and } \\
\text { PDF downloads }\end{array}$ & $\begin{array}{l}\text { No statistically signifi- } \\
\text { cant difference in 6-day } \\
\text { or 30-day page views } \\
\text { (and downloads) }\end{array}$ \\
\hline $\begin{array}{l}\text { Hoang, } \\
2015 \text { [52] }\end{array}$ & Radiology & $\begin{array}{l}2 \text { research articles ap- } \\
\text { pearing on the Ameri- } \\
\text { can Journal of Neuro- } \\
\text { radiology and the } \\
\text { American Journal of } \\
\text { Roentgenology (2013- } \\
2014 \text { ) }\end{array}$ & $\begin{array}{l}\text { Quasi-experimen- } \\
\text { tal (retrospective } \\
\text { cohort) }\end{array}$ & $\begin{array}{l}\text { Blog posts on Radiope- } \\
\text { dia.org; podcast shared on } \\
\text { Twitter and Facebook }\end{array}$ & $\begin{array}{l}\text { HTML views and } \\
\text { PDF downloads }\end{array}$ & $\begin{array}{l}\text { Increased page views } \\
\text { during the intervention; } \\
\text { no increased activity be- } \\
\text { yond the podcast }\end{array}$ \\
\hline $\begin{array}{l}\text { Thoma, } \\
2018[51]\end{array}$ & $\begin{array}{l}\text { Emergency } \\
\text { medicine }\end{array}$ & $\begin{array}{l}29 \text { articles selected for } \\
\text { intervention and con- } \\
\text { trol from the Canadi- } \\
\text { an Journal of Emer- } \\
\text { gency Medicine } \\
(2016)\end{array}$ & $\begin{array}{l}\text { Experimental } \\
(\mathrm{RCT})\end{array}$ & $\begin{array}{l}\text { Podcast or infographic or } \\
\text { standard social media pro- } \\
\text { motion through Twitter and } \\
\text { Facebook }\end{array}$ & HTML views & $\begin{array}{l}\text { Using podcasts and info- } \\
\text { graphics was associated } \\
\text { with increased Altmetric } \\
\text { scores and abstract views } \\
\text { but not full-text article } \\
\text { views; they did not signif- } \\
\text { icantly increase full-text } \\
\text { readership }\end{array}$ \\
\hline $\begin{array}{l}\text { Tonia, } \\
2016[49]\end{array}$ & Public health & $\begin{array}{l}130 \text { articles, } 65 \text { inter- } \\
\text { vention and } 65 \text { con- } \\
\text { trol, from the Interna- } \\
\text { tional Journal of Pub- } \\
\text { lic Health }\end{array}$ & $\begin{array}{l}\text { Experimental } \\
\text { (RCT) }\end{array}$ & $\begin{array}{l}\text { International Journal of } \\
\text { Public Health blog, Twitter, } \\
\text { and Facebook accounts dis- } \\
\text { semination }\end{array}$ & $\begin{array}{l}\text { Article abstract, } \\
\text { PDF views, and } \\
\text { downloads; cita- } \\
\text { tions; AAS }\end{array}$ & $\begin{array}{l}\text { Number of downloads } \\
\text { and the number of cita- } \\
\text { tions significantly corre- } \\
\text { lated for all papers, with } \\
\text { the correlation being } \\
\text { stronger in the interven- } \\
\text { tion group }\end{array}$ \\
\hline
\end{tabular}

\footnotetext{
${ }^{\mathrm{a} A A S}$ : Altmetrics attention score.

${ }^{\mathrm{b}} \mathrm{RCT}$ : randomized controlled trial.
} 
Table 2. Characteristics of correlational studies of very good quality $(n=7)$.

\begin{tabular}{|c|c|c|c|c|c|c|c|}
\hline \multirow[t]{2}{*}{$\begin{array}{l}\text { Study ID and } \\
\text { reference }\end{array}$} & \multirow[t]{2}{*}{$\begin{array}{l}\text { Health research area/unit and period of } \\
\text { analysis }\end{array}$} & \multirow[t]{2}{*}{ Metrics reported } & \multirow[t]{2}{*}{ Results } & \multicolumn{4}{|c|}{$\begin{array}{l}\text { Methodological quali- } \\
\text { ty indicators }\end{array}$} \\
\hline & & & & 1 & 2 & 3 & 4 \\
\hline $\begin{array}{l}\text { Costas, } 2015 \\
{[61]}\end{array}$ & $\begin{array}{l}\text { Biomedical and health sciences; } 217,115 \\
\text { articles in health sciences available from } \\
\mathrm{WoS}^{\mathrm{b}}(2011-2013)\end{array}$ & $\begin{array}{l}\text { Social media: Altmetrics-Biblio- } \\
\text { metrics: Citations }\left(\mathrm{WoS}^{\mathrm{b}}\right)\end{array}$ & $\begin{array}{l}\text { Positive relationship between } \\
\text { number of Altmetrics and the } \\
\text { average citation impact and cita- } \\
\text { tion scores }\end{array}$ & + & + & + & + \\
\hline $\begin{array}{l}\text { Delli, } 2017 \\
\text { [64] }\end{array}$ & $\begin{array}{l}\text { Dental medicine; } 100 \text { articles with high- } \\
\text { est } \mathrm{AAS}^{\mathrm{c}} \text { from Altmetric Explorer and } \\
\mathrm{JCR}^{\mathrm{d}}(2015)\end{array}$ & $\begin{array}{l}\text { Social media: Altmetrics-Biblio- } \\
\text { metrics: Citations (Scopus) }\end{array}$ & $\begin{array}{l}\text { No significant correlation be- } \\
\text { tween Altmetrics and citations }\end{array}$ & + & + & + & + \\
\hline $\begin{array}{l}\text { Eysenbach, } \\
2011[17]\end{array}$ & $\begin{array}{l}\text { Medical informatics; } 208 \text { tweets includ- } \\
\text { ing links to } 286 J M I R^{\mathrm{e}} \text { articles (2008- } \\
2010 \text { ) }\end{array}$ & $\begin{array}{l}\text { Social media: Twitter-Biblio- } \\
\text { metrics: Citations (Google } \\
\text { Scholar and Scopus) }\end{array}$ & Moderate correlations & + & ++ & + & ++ \\
\hline $\begin{array}{l}\text { Haustein, } \\
2014[16]\end{array}$ & $\begin{array}{l}\text { Biomedical and health sciences; } \\
1,431,576 \text { biomedical and health sci- } \\
\text { ences articles available on PubMed } \\
(2010-2012)\end{array}$ & $\begin{array}{l}\text { Social media: Twitter; Altmet- } \\
\text { rics-Bibliometrics: Citations } \\
\left(\mathrm{WoS}^{\mathrm{b}}\right)\end{array}$ & Moderate correlations & + & + & + & + \\
\hline $\begin{array}{l}\text { Knight, } 2014 \\
{[35,36]}\end{array}$ & $\begin{array}{l}\text { Organ transplantation; } 6979 \text { articles with } \\
\text { citation data available; } 1346 \text { with social } \\
\text { media mention (2011-2012) }\end{array}$ & $\begin{array}{l}\text { Social media: Altmetrics-Biblio- } \\
\text { metrics: Citations (Scopus) }\end{array}$ & $\begin{array}{l}\text { Significant correlations between } \\
\text { social media mentions and cita- } \\
\text { tions }\end{array}$ & + & + & + & + \\
\hline $\begin{array}{l}\text { Livas, } 2018 \\
{[71]}\end{array}$ & $\begin{array}{l}\text { Orthodontics; Top } 200 \text { articles in or- } \\
\text { thodontics available from Altmetrics } \\
\text { Explorer (2017) }\end{array}$ & $\begin{array}{l}\text { Social media: Altmetrics-Biblio- } \\
\text { metrics: Citations (Scopus) }\end{array}$ & $\begin{array}{l}\text { No correlation was observed be- } \\
\text { tween Altmetrics score and cita- } \\
\text { tions }\end{array}$ & + & + & + & + \\
\hline $\begin{array}{l}\text { Maggio, } 2018 \\
{[72]}\end{array}$ & $\begin{array}{l}\text { Health profession education; } 2486 \text { arti- } \\
\text { cles with Altmetrics published in health } \\
\text { profession education (2013-2015) }\end{array}$ & $\begin{array}{l}\text { Social media: Altmetrics-Biblio- } \\
\text { metrics: Citations }\left(\mathrm{WoS}^{\mathrm{b}}\right)\end{array}$ & $\begin{array}{l}\text { Significant correlations between } \\
\text { Altmetrics and bibliometrics, but } \\
\text { moderate effects }\end{array}$ & + & + & + & + \\
\hline
\end{tabular}

${ }^{\mathrm{a}} 1$ : appropriately adjusting for time of the social media metric $(+) ; 2$ : appropriately adjusting for confounders such as article type $(+)$ and seasonality/time factors $(++)$; 3 : appropriately exploring correlations by including scatterplots $(+) ; 4$ : appropriately reporting nonlinear correlations tests and statistics $(+)$ as well as log-linear relationship tests $(++)$.

${ }^{\mathrm{b}}$ WoS: Web of Science.

${ }^{\mathrm{c}}$ AAS: Altmetrics attention score.

dJCR: Journal Citation Reports.

e JMIR: Journal of Medical Internet Research. 
Table 3. Characteristics of correlational studies of good quality $(n=8)$.

\begin{tabular}{|c|c|c|c|c|c|c|c|}
\hline \multirow[t]{2}{*}{$\begin{array}{l}\text { Study ID and } \\
\text { reference }\end{array}$} & \multirow[t]{2}{*}{$\begin{array}{l}\text { Health research area/unit and period of } \\
\text { analysis }\end{array}$} & \multirow[t]{2}{*}{ Metrics reported } & \multirow[t]{2}{*}{ Results } & \multicolumn{4}{|c|}{$\begin{array}{l}\text { Methodological quali } \\
\text { ty indicators }{ }^{\mathrm{a}}\end{array}$} \\
\hline & & & & 1 & 2 & 3 & 4 \\
\hline $\begin{array}{l}\text { Dal-Ré, } 2017 \\
\text { [62] }\end{array}$ & $\begin{array}{l}\text { Medical sciences; } 410 \text { original investiga- } \\
\text { tions and } 182 \text { opinion articles published } \\
\text { in the first } 4 \text { printed issues of } 4 \text { top- } \\
\text { ranked general medicine journals and } 1 \\
\text { top-ranked journal on } 5 \text { different medical } \\
\text { specialties that provide Altmetric scores } \\
(2015-2016)\end{array}$ & $\begin{array}{l}\text { Social media: Altmetrics-Biblio- } \\
\text { metrics: Citations (Google } \\
\text { Scholar) }\end{array}$ & $\begin{array}{l}\text { AAS }{ }^{\mathrm{b}} \text { was strongly/moderately } \\
\text { associated with citation count }\end{array}$ & + & + & - & + \\
\hline $\begin{array}{l}\text { Haustein, } \\
2015[66]\end{array}$ & $\begin{array}{l}\text { Biomedical and health sciences; } \\
1,339,297 \text { articles, of which } 595,254 \text { in } \\
\text { biomedical and health sciences, available } \\
\text { from } \operatorname{WoS}^{c}(2012)\end{array}$ & $\begin{array}{l}\text { Social media: Altmetrics-Biblio- } \\
\text { metrics: Citations }\left(\mathrm{WoS}^{\mathrm{c}}\right)\end{array}$ & $\begin{array}{l}\text { No significant correlation be- } \\
\text { tween Altmetrics and citations }\end{array}$ & - & + & + & + \\
\hline $\begin{array}{l}\text { Jabaley, } 2018 \\
{[67]}\end{array}$ & $\begin{array}{l}\text { Sepsis research; Top } 50 \text { articles available } \\
\text { on PubMed (via query; 2012-2017) }\end{array}$ & $\begin{array}{l}\text { Social media: Altmetrics-Biblio- } \\
\text { metrics: Citations (Scopus and } \\
W_{o S}{ }^{c} \text { ) }\end{array}$ & $\begin{array}{l}\text { Weak to moderate correlations } \\
\text { between Altmetrics and citations }\end{array}$ & + & + & + & - \\
\hline $\begin{array}{l}\text { O’Connor, } \\
2017[39,40]\end{array}$ & $\begin{array}{l}\text { Urology; Top } 5 \text { articles of top } 10 \text { jour- } \\
\text { nals in urology (2014-2015) }\end{array}$ & $\begin{array}{l}\text { Social media: Altmetrics-Biblio- } \\
\text { metrics: Citations (Scopus) }\end{array}$ & $\begin{array}{l}\text { Weak positive correlation be- } \\
\text { tween Altmetric score and cita- } \\
\text { tions }\end{array}$ & - & + & + & + \\
\hline $\begin{array}{l}\text { Rosenkrantz, } \\
2017 \text { [76] }\end{array}$ & $\begin{array}{l}\text { Radiology; } 892 \text { articles from selected } \\
\text { radiology journals (2013) }\end{array}$ & $\begin{array}{l}\text { Social media: Altmetrics-Biblio- } \\
\text { metrics: Citations }\left(\mathrm{WoS}^{\mathrm{c}}\right)\end{array}$ & $\begin{array}{l}\text { Significant but weak correlation } \\
\text { between the citation count and } \\
\text { both the AAS }{ }^{\text {b and the number }} \\
\text { of Twitter mentions }\end{array}$ & - & + & + & + \\
\hline $\begin{array}{l}\text { Scotti, } 2017 \\
{[78]}\end{array}$ & $\begin{array}{l}\text { Not specified, hospital; } 268 \text { articles with } \\
\text { Altmetric score out of } 646 \text { articles pub- } \\
\text { lished in } 2013 \text { in indexed journals (with } \\
\text { a } 2012 \mathrm{IF}^{\mathrm{d}} \text { score) by researchers affiliat- } \\
\text { ed to the authors' hospital (2013) }\end{array}$ & $\begin{array}{l}\text { Social media: Altmetrics-Biblio- } \\
\text { metrics: Citations }\left(\mathrm{WoS}^{\mathrm{c}}\right)\end{array}$ & $\begin{array}{l}\text { Altmetrics significantly associat- } \\
\text { ed with } \mathrm{IF}^{\mathrm{d}} \text { as well as Facebook, } \\
\text { Twitter, and Mendeley }\end{array}$ & - & + & + & + \\
\hline $\begin{array}{l}\text { Thelwall, } \\
2013 \text { [81] }\end{array}$ & $\begin{array}{l}\text { Not specified; } 171-135,331 \text { articles with } \\
\text { nonzero Altmetric score and a valid } \\
\text { PubMed ID (2011) }\end{array}$ & $\begin{array}{l}\text { Social media: Altmetrics-Biblio- } \\
\text { metrics: Citations }\left(\mathrm{WoS}^{\mathrm{c}}\right)\end{array}$ & $\begin{array}{l}\text { Significant correlations between } \\
\text { most Altmetrics and citations }\end{array}$ & + & + & - & ++ \\
\hline $\begin{array}{l}\text { Thelwall, } \\
2016 \text { [82] }\end{array}$ & $\begin{array}{l}\text { Medical sciences; } 290,282 \text { articles from } \\
45 \text { fields in Scopus Medicine (2009) }\end{array}$ & $\begin{array}{l}\text { Social media: Altmetrics-Biblio- } \\
\text { metrics: Citations (Scopus) }\end{array}$ & $\begin{array}{l}\text { Significant correlations between } \\
\text { Mendeley readers and citations }\end{array}$ & + & + & - & + \\
\hline
\end{tabular}

${ }^{\mathrm{a}} 1$ : appropriately adjusting for time of the social media metric $(+) ; 2$ : appropriately adjusting for confounders such as article type (+) and seasonality/time factors (++); 3: appropriately exploring correlations by including scatterplots $(+) ; 4$ : appropriately reporting nonlinear correlations tests and statistics $(+)$ as well as log-linear relationship tests $(++)$.

${ }^{\mathrm{b}}$ AAS: Altmetrics attention score.

${ }^{\mathrm{c}}$ WoS: Web of Science.

${ }^{\mathrm{d}} \mathrm{IF}$ : impact factor. 
Table 4. Characteristics of correlational studies of fair quality $(n=10)$.

\begin{tabular}{|c|c|c|c|c|c|c|c|}
\hline \multirow[t]{2}{*}{$\begin{array}{l}\text { Study ID and } \\
\text { reference }\end{array}$} & \multirow[t]{2}{*}{$\begin{array}{l}\text { Health research area/unit and period of } \\
\text { analysis }\end{array}$} & \multirow[t]{2}{*}{ Metrics reported } & \multirow[t]{2}{*}{ Results } & \multicolumn{4}{|c|}{$\begin{array}{l}\text { Methodological quali- } \\
\text { ty indicators }\end{array}$} \\
\hline & & & & 1 & 2 & 3 & 4 \\
\hline $\begin{array}{l}\text { Araujo, } 2018 \\
{[54]}\end{array}$ & $\begin{array}{l}\text { Physiotherapy; } 200 \text { randomly selected } \\
\text { articles from physiotherapy evidence } \\
\text { database (PEDro; 2013-2016) }\end{array}$ & $\begin{array}{l}\text { Social media: Altmetrics men- } \\
\text { tioned/reader-Bibliometrics: } \\
\text { Citations }\left(W_{o} S^{b}\right)\end{array}$ & $\begin{array}{l}\text { Significant correlation with cita- } \\
\text { tions }\end{array}$ & + & + & - & - \\
\hline $\begin{array}{l}\text { Calopedos, } \\
2017[58]\end{array}$ & $\begin{array}{l}\text { Urology; } 22 \text { urology articles in English } \\
\text { language identified via PubMed (2010- } \\
\text { 2015) }\end{array}$ & $\begin{array}{l}\text { Social media: Altmetrics-Biblio- } \\
\text { metrics: Citations (Google } \\
\text { Scholar) }\end{array}$ & $\begin{array}{l}\text { Significant correlation between } \\
\text { Altmetrics and citations }\end{array}$ & + & - & + & - \\
\hline $\begin{array}{l}\text { Chang, } 2019 \\
{[59]}\end{array}$ & $\begin{array}{l}\text { Pediatric surgery; } 140 \text { articles appearing } \\
\text { on } 14 \text { core journals on pediatric surgery } \\
(2012-2015)\end{array}$ & $\begin{array}{l}\text { Social media: Altmetrics-Biblio- } \\
\text { metrics: Citations (Scopus); IF } \\
\left(\mathrm{JCR}^{\mathrm{d}}\right)\end{array}$ & $\begin{array}{l}\text { Strong correlations between Alt- } \\
\text { metric scores and citations, but } \\
\text { not } \mathrm{IF}^{\mathrm{c}}\end{array}$ & + & - & + & - \\
\hline $\begin{array}{l}\text { Dardas, } 2019 \\
{[63]}\end{array}$ & $\begin{array}{l}\text { Nursing; } 100 \text { articles in nursing with } \\
\text { highest } \mathrm{AAS}^{\mathrm{e}} \text { from } \mathrm{WoS}^{\mathrm{b}}(2012-2018)\end{array}$ & $\begin{array}{l}\text { Social media: Altmetrics-Biblio- } \\
\text { metrics: Citations }\left(\mathrm{WoS}^{\mathrm{b}} \text { and }\right. \\
\text { Scopus) }\end{array}$ & $\begin{array}{l}\text { Significant moderate correlation } \\
\text { between Altmetrics and citation } \\
\text { counts }\end{array}$ & - & + & + & - \\
\hline $\begin{array}{l}\text { Hassona, } 2019 \\
{[65]}\end{array}$ & $\begin{array}{l}\text { Dental medicine; } 100 \text { articles with high- } \\
\text { est AAS }{ }^{\mathrm{e}} \text { from Altmetric Explorer } \\
(2018)\end{array}$ & $\begin{array}{l}\text { Social media: Altmetrics-Biblio- } \\
\text { metrics: Citations (Google } \\
\text { Scholar and Scopus) }\end{array}$ & $\begin{array}{l}\text { No significant correlation be- } \\
\text { tween Altmetrics and citations }\end{array}$ & - & + & - & + \\
\hline Liu, 2013 [70] & $\begin{array}{l}\text { Field not specified; } 33,128 \text { articles ap- } \\
\text { pearing in PLOS One (2011) }\end{array}$ & $\begin{array}{l}\text { Social media: Altmetrics-Biblio- } \\
\text { metrics: HTML views, PDF } \\
\text { downloads, and citations (Sco- } \\
\text { pus, PubMed, and CrossRef) }\end{array}$ & $\begin{array}{l}\text { Significant correlations between } \\
\text { Altmetrics and bibliometrics }\end{array}$ & - & - & + & + \\
\hline $\begin{array}{l}\text { Nolte, } 2019 \\
{[37,38]}\end{array}$ & $\begin{array}{l}\text { Urology; } 44 \text { articles tweeted about the } \\
2015 \text { American Urological Association } \\
\text { meeting (2015) }\end{array}$ & $\begin{array}{l}\text { Social media: Twitter-Biblio- } \\
\text { metrics: } \text { IF }^{\mathrm{c}}\end{array}$ & $\begin{array}{l}\text { Positive significant correlation } \\
\text { with subsequent publication } \mathrm{IF}^{\mathrm{c}} \\
\text { within } 18 \text { months of presentation }\end{array}$ & - & + & + & - \\
\hline $\begin{array}{l}\text { Punia, } 2019 \\
\text { [73] }\end{array}$ & $\begin{array}{l}\text { Neurological research; Top } 100 \text { articles } \\
\text { from top } 5 \text { neurology journals (2016) }\end{array}$ & $\begin{array}{l}\text { Social media: Altmetrics-Biblio- } \\
\text { metrics: Citations }\end{array}$ & $\begin{array}{l}\text { Weak positive correlation be- } \\
\text { tween Altmetric score and cita- } \\
\text { tions }\end{array}$ & - & - & + & + \\
\hline $\begin{array}{l}\text { Quintana, } \\
2016[74]\end{array}$ & $\begin{array}{l}\text { Psychiatry; } 438 \text { articles in the American } \\
\text { Journal of Psychiatry (2013-2015) }\end{array}$ & $\begin{array}{l}\text { Social media: Twitter-Biblio- } \\
\text { metrics: Citations }\left(\mathrm{WoS}^{\mathrm{b}}\right)\end{array}$ & $\begin{array}{l}\text { Positive correlation between } \\
\text { Twitter mentions and citations }\end{array}$ & + & - & - & ++ \\
\hline $\begin{array}{l}\text { Ruano, } 2018 \\
{[25]}\end{array}$ & $\begin{array}{l}\text { Psoriasis research; } 164 \text { systematic re- } \\
\text { views or meta-analyses available from } \\
\text { MEDLINE }^{\text {f }} \text { EMBASE }^{\mathrm{g}} \text {, and Cochrane } \\
\text { databases (2016) }\end{array}$ & $\begin{array}{l}\text { Social media: Altmetrics-Biblio- } \\
\text { metrics: Citations (Google } \\
\text { Scholar) }\end{array}$ & $\begin{array}{l}\text { No significant correlation be- } \\
\text { tween Altmetrics and citations; } \\
\text { The number of Mendeley readers } \\
\text { was significantly associated with } \\
\text { citations }\end{array}$ & - & + & + & - \\
\hline
\end{tabular}

${ }^{\mathrm{a}} 1$ : appropriately adjusting for time of the social media metric $(+) ; 2$ : appropriately adjusting for confounders such as article type $(+)$ and seasonality/time factors $(++)$; 3 : appropriately exploring correlations by including scatterplots $(+)$; 4 : appropriately reporting nonlinear correlations tests and statistics $(+)$ as well as log-linear relationship tests $(++)$.

${ }^{b_{W o S}}$ : Web of Science.

${ }^{\mathrm{C}} \mathrm{IF}$ : impact factor.

dCR: Journal Citation Reports.

eAAS: Altmetrics attention score.

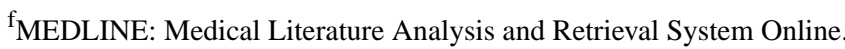

${ }^{g}$ EMBASE: Excerpta Medica database. 
Table 5. Characteristics of correlational studies of poor quality $(n=12)$.

Study ID and Health research area/unit and period of Metrics reported reference analysis
Results Methodological quali-

ty indicators ${ }^{\mathrm{a}}$

$\begin{array}{llll}1 & 2 & 3 & 4\end{array}$

Amath, 2017 Medical education: 482 articles appear- Social media: Twitter, Mende[32,33] ing on Medical Education journal (2012- ley; Altmetrics-Bibliometrics: 2013) Citations (Scopus)
Azer, 2019

Batooli, 2016 Medical sciences; 533 articles published by faculty at Kashan University of Medical Sciences (1997-2014)

articles in medical professionalism identified by searching WoS ${ }^{\mathrm{b}}$ (19942011)

Transplantation; All articles published on transplantation in 2015 (volume 99)

Social media: Twitter-Bibliometrics: number of views and downloads

Social media: Altmetrics-Bibliometrics: Citations $\left(\mathrm{WoS}^{\mathrm{b}}\right.$ )

Social media: ResearchGate, Mendeley-Bibliometrics: Citations (Scopus)
Very strong correlation between

Tweet counts and Altmetrics score; Citations were strongly correlated with access counts and Mendeley downloads, and weakly and moderately correlated respectively with Twitter mentions and Altmetric scores

No significant correlation between Altmetrics and citations

Significant correlation between downloads and Twitter activity

Positive correlation between the number of views of articles in ResearchGate and citations; positive correlation between reading frequency in Mendeley and citations; number of views of articles in ResearchGate correlated with higher reading frequency in Mendeley and citations

Chen, 2019 Rheumatology; 1460 articles appearing [41] in Rheumatology journal (2010-2015)

Social media: Altmetrics-Bibliometrics: Citations and downloads

Strong correlations between Altmetric and downloads, but not citations

No significant correlation between Twitter and citations metrics: Citations (Google Scholar)

Chiang, 2016 Gastroenterology; 1671 articles appear- Social media: Twitter-Bit [42] 482 being tweeted (2012)

Social media: ImpactStory;

Cho, 2017 Medical sciences; 98 articles from medical sciences from Korean researchers in Scopus (2010-2014)

Altmetrics-Bibliometrics: Citations (Scopus)

The more the papers are cited in the journal, the more papers saved on Mendeley

Hayon, 2019 Urology; 213 articles from 7 prominent [34] urology journals (2014-2015)

Social media: Altmetrics-Bibliometrics: Citations (Google Scholar and Scopus)

Positive relationship between Twitter activity and Scopus citations Social media: Twitter-Biblio-

Jedhav, 2019 Neurointerventional surgery; 451 articles [68] first published on the web on the Journal of Neurointerventional Surgery (20152016) metrics: Citations $\left(\mathrm{WoS}^{\mathrm{b}}\right)$

The level of evidence of the publication and the topic of research strongly predicts future citations. The number of clicks also appears to be a strong predictor of future citations, and the number of clicks increases as the number of Twitter users also grows

Significant correlations between $-\quad+\quad-\quad-$ citations and Twitter activity metrics: Citations $\left(\mathrm{WoS}^{\mathrm{b}}\right.$ )

[69] on 3 journals with Twitter profiles (2015-2016)

Konstantiniuk, Sepsis research; 12 articles on sepsis 2015 [44] compared with 8 articles on $\mathrm{ICU}^{\mathrm{c}}$ (period not indicated)

Social media: Twittter; Altmetrics; ResearchGate-Bibliometrics: Citations (Google Scholar and $\mathrm{WoS}^{\mathrm{b}}$ )

Shirazi, 2018 Health literacy; 615 articles with a digital [79] object identifier and indexed in $\mathrm{WoS}^{\mathrm{b}}$ (2015)
Social media: Altmetrics-Bibliometrics: Citations $\left(\mathrm{WoS}^{\mathrm{b}}\right)$
The Altmetric score neither correlated with Google Citations nor publishing date

Significant correlations between - $\quad$ - $\quad$ - + Altmetrics and citations 
${ }^{\mathrm{a}} 1$ : appropriately adjusting for time of the social media metric (+); 2 : appropriately adjusting for confounders such as article type (+) and seasonality/time factors $(++)$; 3 : appropriately exploring correlations by including scatterplots $(+)$; 4: appropriately reporting nonlinear correlations tests and statistics $(+)$ as well as log-linear relationship tests (++).

${ }^{\mathrm{b}}$ WoS: Web of Science.

${ }^{\mathrm{c}} \mathrm{ICU}$ : Intensive Care Unit.

Table 6. Characteristics of correlational studies of very poor quality $(\mathrm{n}=7)$.

\begin{tabular}{|c|c|c|c|c|c|c|c|}
\hline \multirow[t]{2}{*}{$\begin{array}{l}\text { Study ID and } \\
\text { reference }\end{array}$} & \multirow[t]{2}{*}{$\begin{array}{l}\text { Health research area/unit and period of } \\
\text { analysis }\end{array}$} & \multirow[t]{2}{*}{ Metrics reported } & \multirow[t]{2}{*}{ Results } & \multicolumn{4}{|c|}{$\begin{array}{l}\text { Methodological quali- } \\
\text { ty indicators }{ }^{\mathrm{a}}\end{array}$} \\
\hline & & & & 1 & 2 & 3 & 4 \\
\hline $\begin{array}{l}\text { Araujo, } 2017 \\
{[53]}\end{array}$ & $\begin{array}{l}\text { Parkinson disease research; Top } 20 \text { arti- } \\
\text { cles with highest AAS }{ }^{\mathrm{b}} \text { appearing on the } \\
\text { Journal of Parkinson's Disease (2013- } \\
\text { 2016) }\end{array}$ & $\begin{array}{l}\text { Social media: Twitter, Face- } \\
\text { book, Altmetrics-Bibliometrics: } \\
\text { Citations (Scopus) }\end{array}$ & $\begin{array}{l}\text { Qualitative summary in support } \\
\text { of correlation }\end{array}$ & - & - & - & - \\
\hline $\begin{array}{l}\text { Heydarpour, } \\
2017[43]\end{array}$ & $\begin{array}{l}\text { Multiple sclerosis research; } 4693 \text { articles } \\
\text { on multiple sclerosis retrieved from Alt- } \\
\text { metric Explorer and PubMed (2016) }\end{array}$ & $\begin{array}{l}\text { Social media: Altmetrics-Biblio- } \\
\text { metrics: Citations }\left(\mathrm{WoS}^{\mathrm{c}}\right)\end{array}$ & $\begin{array}{l}\text { Moderate correlations between } \\
\text { AAS }^{\text {b }} \text { and citations }\end{array}$ & - & - & - & - \\
\hline $\begin{array}{l}\text { Matava, } 2017 \\
{[45]}\end{array}$ & $\begin{array}{l}\text { Pediatric anesthesiology; Top } 100 \text { arti- } \\
\text { cles on pediatric anesthesiology available } \\
\text { from Altmetrics Explorer (2016) }\end{array}$ & $\begin{array}{l}\text { Social media: Altmetrics-Biblio- } \\
\text { metrics: Citations (Scopus) }\end{array}$ & $\begin{array}{l}\text { No significant correlation be- } \\
\text { tween Altmetrics or Twitter } \\
\text { mentions and Citations; The } \\
\text { number of Mendeley mentions } \\
\text { was significantly associated with } \\
\text { citations }\end{array}$ & - & - & - & - \\
\hline $\begin{array}{l}\text { Ramezani- } \\
\text { Pakpour- } \\
\text { Langeroudi, } \\
2018 \text { [75] }\end{array}$ & $\begin{array}{l}\text { Clinical medicine; } 55 \text { highly cited arti- } \\
\text { cles on Thomson Reuters' Essential Sci- } \\
\text { ence Indicator (2015) }\end{array}$ & $\begin{array}{l}\text { Social media: ResearchGate, } \\
\text { Mendeley, Academia, } \\
\text { LinkedIn-Bibliometrics: Cita- } \\
\text { tions (Scopus) }\end{array}$ & $\begin{array}{l}\text { A positive direct relationship was } \\
\text { observed between visibility at } \\
\text { social networking sites with cita- } \\
\text { tion and } \mathrm{h} \text { - index rate }\end{array}$ & - & - & - & - \\
\hline $\begin{array}{l}\text { Ruan, } 2018 \\
{[77]}\end{array}$ & $\begin{array}{l}\text { Plastic and reconstructive surgery; } 55 \\
\text { most-cited articles published in Plastic } \\
\text { and Reconstructive Surgery (2014-2015) }\end{array}$ & $\begin{array}{l}\text { Social media: Altmetrics, } \\
\text { Mendeley-Bibliometrics: Cita- } \\
\text { tions (Scopus) }\end{array}$ & $\begin{array}{l}\text { No significant correlation be- } \\
\text { tween Altmetrics and citations; } \\
\text { The number of Mendeley men- } \\
\text { tions was significantly associated } \\
\text { with citations }\end{array}$ & - & - & - & - \\
\hline $\begin{array}{l}\text { Smith, } 2019 \\
{[80]}\end{array}$ & $\begin{array}{l}\text { Gastrointestinal endoscopy; } 2361 \text { origi- } \\
\text { nal research articles published in Gas- } \\
\text { trointestinal Endoscopy of which } 2050 \\
\text { were cited at least once (2010-2016) }\end{array}$ & $\begin{array}{l}\text { Social media: Altmetrics-Biblio- } \\
\text { metrics: Citations (Scopus) }\end{array}$ & $\begin{array}{l}\text { Significant correlations between } \\
\text { tweets and citations }\end{array}$ & - & - & - & - \\
\hline $\begin{array}{l}\text { Wiehn, } 2017 \\
\text { [83] }\end{array}$ & $\begin{array}{l}\text { Medical sciences; } 36 \text { Shire-sponsored } \\
\text { articles (2016) }\end{array}$ & $\begin{array}{l}\text { Social media: Altmetrics-Biblio- } \\
\text { metrics: Article downloads and } \\
\mathrm{IF}^{\mathrm{d}}\end{array}$ & $\begin{array}{l}\text { No correlation was observed be- } \\
\text { tween Altmetrics score and IF } \\
\text { downloads }\end{array}$ & - & - & - & - \\
\hline
\end{tabular}

${ }^{\mathrm{a}} 1$ : appropriately adjusting for time of the social media metric $(+) ; 2$ : appropriately adjusting for confounders such as article type $(+)$ and seasonality/time factors $(++)$; 3 : appropriately exploring correlations by including scatterplots $(+)$; 4 : appropriately reporting nonlinear correlations tests and statistics $(+)$ as well as log-linear relationship tests $(++)$.

${ }^{\mathrm{b}}$ AAS: Altmetrics attention score.

${ }^{\mathrm{c}}$ WoS: Web of Science.

${ }^{\mathrm{d}}$ IF: impact factor.

\section{Is There Evidence of the Impact of Social Media?}

In this section we elaborated on the findings related to the different areas of research, study type, social media intervention characteristics, metrics assessed, and reported results, as summarized in Table 1.

\section{Areas of Health Research}

Impact studies have reported the use of social media interventions to promote original research articles published in academic journals in the subject areas of clinical pain sciences (ie, PLOS ONE) [48], cardiology (ie, Circulation) [46,47], radiology [52], emergency health [51] public health (ie, International Journal of Public Health) [49], and women's health (various journals) [50].

\section{Study Types}

We identified 4 randomized controlled experiments, further referred to as $R C T s$ [ $46,47,49,51]$, and 3 quasi-experimental trials $[48,50,52]$.

\section{Social Media Intervention Characteristics}

Articles in the intervention conditions were shared on Twitter or Facebook social media profiles of the targeted journals using 
automated or manually made posts leveraging the organic (ie, unpaid) reach of each social networking site. One study used advertising to increase the views of Facebook posts (ie, boosted content) to increase reach, which is the number of viewers of the post [47]. Intervention duration ranged from 12 [49], 18 [48], 34 [47], to 52 weeks [46]. The intensity varied considerably: only once the content was blogged [48], 1 post every 2 weeks and 12 weeks [49], 1 or 2 posts per week [46], or several posts per day [47]. Three studies used blogs to diffuse the studies further [48,49,52]. Thoma et al [51] tested the use of a podcast and infographics as complementary information in addition to Facebook and Twitter content sharing. The interventions varied in nature of the message posted, the profile of the social media owner(s), the duration of the posting, its intensity, and whether an incentive or paid promotion was used. For example, in one of the quasi-experimental trials [48], the authors advertised the posts related to 16 original research articles about clinical pain sciences, published on PLOS ONE, through a systematic intervention targeting Facebook, Twitter, LinkedIn, and ResearchBlogging.org. The authors used a systematic protocol (ie, timing the frequency of release of the messages) to direct social media users to read a web-based version of the original research article.

\section{Metrics Assessed}

These studies investigated the effect of social media interventions on subsequent access to web-based journal content and article downloads. Two studies (1 experimental [49] and 1 quasi-experimental [48]) reported the effect on the number of citations.

\section{Reported Results}

Although the quasi-experimental trials reported an increase in HTML and PDF views during the intervention [48,50,52], the RCTs reported no significant changes in the same metrics $[46,47,49,51]$. There were no significant effects on the number of citations in both the quasi-experiment [48] and the RCT [49].

\section{What Is the Association Between Altmetrics and Bibliometrics in Health Research?}

In this section we present the results related to the correlational studies identified.

\section{Areas of Health Research}

The 44 correlational studies evaluated the relationship between Altmetrics and bibliometrics in a variety of health research fields and disciplines, covering generic medical or biomedical research disciplines [16,57,60-62,66,70,78,81-83], or more specific disease-related research fields, such as multiple sclerosis [43], neurological research [73], Parkinson disease [53], psoriasis [25], and sepsis [44,67]. Some articles covered clinical or dental medicine $[64,65,75]$ and different kinds of surgery $[59,68,77]$; others focused on specialized branches of medicine, such as urology [34,38,39,58], radiology [76], and coloproctology [69].

\section{Study Types}

Almost all correlational studies were quantitative cross-sectional studies $(43 / 44,97 \%)$ examining the relationship between bibliometrics and various social media metrics or monitored citation trends over time [52,83]. The association between social media use and citations was also discussed in a qualitative study [53].

\section{Metrics Assessed}

Correlational studies encompassed a wide variety of Altmetrics and bibliometrics. Some correlational studies have investigated the association between Altmetrics scores and citations in Web of Science, Scopus, or Google Scholar $[36,39,53,61,62,66,70,74,76,81-83]$. One study investigated the correlation between ImpactStory indices and Scopus citations [60]. Other studies focused on the usage metrics of specific social media platforms, such as Twitter $[17,42,56,74]$, blogs, web-based posts [52,58], ResearchGate, or Mendeley [57]. A total of 4 studies reported data on the association between the Altmetric of articles and the IF of journals in which they were published [38,59,60,83]; 3 studies investigated the relationship between sharing articles on academic social media platforms, such as ResearchGate and reference management software Mendeley [44,57,75].

\section{Methodological Quality}

As shown in the last 4 columns of Tables 2 to 6 , the methodological quality of the papers varied according to the type of article and amount of detail included in the publication. The 5 conference abstracts included in the list of correlational studies [41-45] did not provide sufficient information to meet most of the methodological quality indicators. Only 3 of these $[41,42,44]$ provided different correlation results according to the type of disease area, topic, or article type; hence, they were deemed to have adjusted for confounders. Of the remaining 39 studies with full-text, 16 adjusted the social media metric for time $(16 / 39,41 \%) ; 22$ studies adjusted for confounders such as article type or topic $(22 / 39,56 \%)$, but none included the seasonality and timeframe of the article publication. Some 21 studies included scatterplots to explore the type of correlation $(21 / 39,54 \%)$, and 18 studies reported the use of Spearman rho or other nonparametric tests when comparing social media citation counts $(18 / 39,46 \%)$. However, only 2 studies [74,81] reported analyses for tests based on ranking similar to log-linear correlations included in a study by Eysenbach [17]. The method used in a study by Quintana and Doan [74], but described in the paper by Thelwall et al [81], "compares a given publication against the publications that appeared immediately before and after it. A successful test occurs when the number of Twitter mentions and citations for a given publication are both higher (or both lower) than the average of Twitter mentions and citations of the two adjacent articles" [81].

A total of 6 of 39 studies (15\%) [16,36,61,64,71,72] met the minimum standard in all 4 criteria mentioned above, but none followed exemplar paper by Eysenbach [17] when reporting correlations between social media metrics and citations. In all, 7 studies scored at least three criteria $(7 / 39,18 \%)$. Of these studies, 4 scored criteria 2-3-4 [40,66,76,78], 2 studies scored 1-2-4 [62,82], and 1 study 1-2-3 [67]. The remaining 18 studies scored less than 2 of any quality criteria $(18 / 39,46 \%)$. 


\section{Reported Results}

Twitter was the most popular social media platform discussed in correlational studies (21 of 44 studies) $[16,17,32,34,38,42,45,53,55,56,59,62,63,68,69,72,74,76,78-80]$, followed by Mendeley (15 studies) $[25,45,55,57,60,63,71,72,75-79,82,83]$. In reference to the association between Altmetric scores and bibliometrics, the results were mixed. No significant correlations were reported in 12 of 44 studies $(27 \%)$ [25,41,42,44,45,55,64-66,71,77,83]; weak or moderate correlations were reported by authors in 13 of 44 studies (30\%) [16,17,39,43,53,61-63,67,72-74,76]. Strong positive associations were reported by the authors of half (22) of the correlational studies. In particular, strong associations were found between Mendeley readership (reads and downloads) and subsequent citations $[25,45,57,60,75,77-79,82]$. This is also consistent with the findings reported in one of the impact studies [49], which found significant correlations between the number of downloads of a paper and the subsequent number of citations $(\mathrm{r}=0.52 ; P<.001)$, which were larger in the intervention group $(\mathrm{r}=0.67 ; \quad P<.001)$. Only 2 studies reported no significant associations between traditional and social media metrics [66] and between Altmetric score and journal IF, ResearchGate reads, and the number of article downloads [83].

When focusing only on the 7 high quality studies (ie, those meeting all methodological quality criteria 1-4) $[16,17,36,61,64,71,72], 5$ studies reported correlation coefficients of moderate size $[16,17,36,61,72]$ and 2 studies, from the same discipline (orthodontics), reported no significant correlations $[64,71]$.

\section{Discussion}

\section{Is There Evidence of the Impact of Social Media?}

The primary aim of this review was to evaluate the effect of social media on the dissemination of health research output. Of the 51 identified studies, only 7 were experimental studies aimed at establishing a causal link between social media use and subsequent citations. The identified impact studies provide suggestive yet inconclusive evidence on the effect of using social media to increase the number of citations, thus contributing to the dissemination of health research according to traditional bibliometric indicators. This result is consistent with findings reported in a systematic review, which was aimed at describing the interactions between bibliometric factors and social media activity on the impact of reviews in the field of psoriasis [25]. The findings suggest that although Google Scholar citations were predicted by the number of readers on Mendeley and year of publication (ie, 2015 and 2016), the number of tweets and the IF of a journal were not. Moreover, a journal's IF was the sole significant predictor of the number of tweets [25]. Careful considerations should be made, as the limited number of studies we identified does not allow us to generate strong conclusions or recommendations.

In our review, we identified four impact studies [46,47,49,51], which used randomized controlled experiments. However, unlike in the TrendMD dissemination study [19], these RCTs did not show consistent effects of the social media dissemination strategies on citations over time. This finding might be due to several limitations. First, the experiments used social media interventions, which had very different intensities (eg, once vs several posts per day) and duration (ie, 12-52 weeks). This fact does not allow us to determine whether the effect was due to a dose-response or to mere exposure to the intervention. Consequently, the long-term effects of social media interventions are still unclear as the only long-term study (52 weeks) [46] reported no difference in median 30-day page views between the intervention and control conditions. However, a larger, longitudinal RCT on TrendMD distribution showed higher citation counts in health and medical science articles after 6 and 12 months than in a control group [19]. Second, the reported social media impact outcomes differed in terms of content and type of social media channel used, frequency and intensity of social media use, type of exposure, and unit of analysis. It is important to note that careful consideration should be made when comparing social media interventions across the spectrum of platforms and types of social networking sites. A journal's or author's Facebook page or Twitter handle that originally has 100,000 followers would very likely increase the reach of an article shared, compared with a page that has only 1000 followers. This finding could have implications on the effect of increased citations in journals that already have a high IF compared with journals that have a low IF (as the accounts of the former would have a higher number of followers). In other words, the social media interventions were too heterogeneous to compare among each another and to perform meta-analyses.

Future impact studies should maintain a rigorous study design; consistently report social media outcomes using standard Altmetric scores; provide better and more detailed explanations about the specific timeframes, exposure, frequency, and intensity of interventions for comparability. In other words, future studies should answer research questions such as to what extent does the frequency of social media posting influence short-term indicators (eg, number of PDF downloads) and long-term indicators (eg, Altmetric score and citations)? To what extent does posting on Mendeley and Twitter, as opposed to Twitter, have an impact on the Altmetric score and citations?

This information could help researchers specialized in systematic reviews to develop accurate evidence, including meta-analyses; the information could also be helpful for researchers aimed at testing different social media intervention strategies or at comparing similar methodologies in different domains or disciplines. Other factors that can explain the lack of findings are in the topics of the information shared on social media. Some very specific health disciplines have limited readership, as they require specific knowledge to understand the content that is shared.

\section{What Is the Association Between Altmetrics and Bibliometrics in Health Research?}

Another finding of this review is that most of the available evidence focuses on describing correlations between traditional and social media metrics in health research. The included 44 correlational studies provide further support that, in general, the higher the AAS, the higher the subsequent citations will be. However, the studies reported wide variability in the magnitude 
of correlation coefficients and provided a variety of interpretations for the strength of these correlations, which warns some caution. Most reports did not provide in-depth evaluations of the correlations, including, for example, confidence intervals of the correlation coefficients; an analysis of the distribution of citation counts and social media metrics, which tend to violate the assumptions of normality; and the use of visual representations such as scatterplots, as recommended by some researchers $[84,85]$. We identified only 6 correlational studies $[16,36,61,64,71,72]$ meeting the minimum methodological quality criteria (1-4) described in the methodology, in addition to the seminal paper by Eysenbach, 2011 [17]. Most notably and quite surprisingly, none of the identified 44 studies followed the recommendations and metrics suggested by Eysenbach. No paper included the Twindex or the Twimpact factor or correctly adjusted the social media metrics for the time since publication or seasonality in publication and for the skewed distribution of the metric, for example, by dichotomizing result or testing for log-linear correlations.

Twitter and Mendeley seem to be the indicators that contribute the most to the Altmetric score. Mendeley and ResearchGate were positively associated with subsequent citations $[25,45,77,79]$. Although Twitter can be used to disseminate research output among a broader, general public, the use of Mendeley and ResearchGate seems to be restricted to specific target audience of researchers or media professionals. Unsurprisingly, the more an article is shared on ResearchGate or Mendeley, the more it will be cited. This finding might indicate that researchers use reference management software (Mendeley) to organize their libraries for research purposes and then share their research on a specialized social networking site (ResearchGate). This software allows users to discover new related research because the platform itself suggests new evidence based on the users' previous reads and mentions. Although this does not imply that research has been disseminated among the wider public, ResearchGate and Mendeley appear fundamental for the research community.

Our findings are consistent with some existing review evidence investigating the domain of medical research output [24], as well as other multidisciplinary research fields [21,22], or marketing [20]. Another review that focused on orthopedic research journals [86] reported that web-based mentions were weakly yet positively related to various bibliometric indices, such as the number of citations, journal IF, Eigenfactors, and h-index values for the first and last authors. In addition, a systematic review of reviews published in scientific journals related to skin psoriasis [25] found an association between Altmetrics and bibliometric indicators. The association between traditional bibliometrics (eg, number of citations) and social media metrics (eg, number of mentions) could be an indicator of a positive effect of using social media on research dissemination. However, not all correlational articles identified showed strong positive correlations. The association could be confounded by several factors, such as the value of the research paper or the popularity of the topic, as we have previously discussed. In other words, a high-value research paper could lead to both high exposure on social media and a high number of citations, depending on the intrinsic subject. An article discussing skin psoriasis, or another more common disease, might attract the attention of the wider general public, compared with coloproctology and neuro-interventional surgery, which attract audiences of specialized health care professionals. Moreover, researchers can discover articles to cite from other traditional sources, such as electronic databases and libraries. Once an article is published and cited, other researchers might want to share the discovery on their social networking sites. Hence, using social media to diffuse health research may generate a virtuous circle that can be beneficial for both individual researchers and journals, as this will result in an increased IF. We need more evidence of good quality experimental, impact studies rather than correlational studies to establish a causal link between social media use and impact on citations. We also need better reporting of correlational studies; following the suggestions included in the seminal paper by Eysenbach [17], researchers should strive to provide more insight (and data) on the actual distribution of bibliometrics and social media metrics analyzed so that more meaningful interpretations of the relationship between these variables could be drawn.

\section{Recommendations}

Scientific impact is a multidimensional concept that cannot be adequately measured by a single indicator [87]. As the AAS suggests, social media impact is even more multidimensional, as it is linked to various web-based tools that a particular researcher can use. This researcher is also embedded in a unit within an institution [88]; the researcher's work is then published in a journal, which has a specific and independent impact (IF, citations, and Altmetrics). The evaluation of the researcher's scientific impact on social media should then take into account various dimensions and indicators and be aligned with more qualitative evaluation on other domains of the RIF [4], which includes policy, service, and societal impacts.

In conclusion, is social media dissemination worth the effort? On the basis of the findings of this review, we recommend researchers in health sciences to continue using social media to disseminate their research, as there is some data suggesting its long-term impact on citations following dissemination on the internet using paid services (eg, TrendMD study) [19]. Researchers should use popular social networking platforms, such as Twitter or Facebook, first to engage with the general public and the media, to design more citizen-oriented research and democratize their findings. Researchers should also use popular social networking sites to interact with peers and discuss their research as well as disseminate their findings. Researchers should also diffuse their work on specialized social networking sites for academics, such as Mendeley, ResearchGate, and Academia. The content shared on these networks is very likely to be cited, as researchers who are on Mendeley may use it as a reference management software. Mendeley and ResearchGate databases may be used in combination with traditional electronic databases for literature reviews and similar activities. Storing copies of articles on these platforms could increase the chances of a paper being cited in the future.

Although more rigorous longitudinal research needs to establish whether social media activity can be linked to increased 
citations, it is important to consider social media as useful tools to reach a wider public, not just specialized audiences. Researchers should put effort into translating knowledge for different target audiences, bearing in mind the users of each social media channel. The AAS can be a useful instrument that allows researchers and institutions to evaluate social media impact by distinguishing among the attention generated by specialized applications (Mendeley and ResearchGate) or by the wider public and traditional media (Facebook, Twitter, and blogs).

\section{Strengths and Limitations}

This review sheds light on the use of social media to disseminate health research output. The main strength of this review is the use of standard methods of a systematic review, including a comprehensive search strategy, a duplicate approach to study selection and data abstraction, and detailed data abstraction. The main limitation is that we were not able to conduct a meta-analysis because of the substantial variability in the included studies. For example, the impact studies varied in terms of the interventions evaluated (eg, type of social media used, the message posted, and its duration and intensity) and the outcomes assessed.

\section{Conclusions}

Our findings have implications for research in the field of health-related metrics. There is a need for more and better designed experimental studies testing the use of social media to increase the dissemination of health research. These studies should be of a randomized design, evaluate the appropriate use of social media, and assess a variety of outcomes (both all Altmetrics dimensions and traditional bibliometrics) over a meaningfully long period. For example, experimental trials could test different strategies to diffuse research articles on social media, by comparing paid (or boosted) content with nonpaid, organic posts. Other trials could include the diffusion of research article links on numerous social networking sites versus a limited number of sites. Once there is evidence of the effectiveness of using social media (as opposed to not using them), different approaches should be investigated.

Our findings also have implications for the practice of using social media for research dissemination. Researchers should not use social media for the sole purpose of increasing their research productivity (ie, number of publications), as there is currently no evidence to support such an effect. They can use it for other purposes, such as disseminating their findings to social media users. When using social media, they have to be attentive to details such as the content of the message, its frequency, and the use of incentives or paid promotions, as this could affect the reach of the posts.

\section{Acknowledgments}

The authors would like to acknowledge Ms Aida Farha from AUB libraries for her help in developing the search strategy and Ms Layal Hneiny, who performed the electronic database search updates. The authors would also like to thank Mr Fadel Al Turki, Dr Sanaa Baddour, Ms Maya Hammoud, and Ms Matilda Sang for their help in reviewing the abstracts.

\section{Authors' Contributions}

EAA, RER, MB, LIM, and SB conceived and designed the review, and EAA, RER, and MB coordinated it. EAA, RER, MB, and LIM were involved in developing the search strategy, and MC, EWA, ASR, and MB extracted the data. Data analyses were undertaken by EAA, RER, and MB, whereas data interpretation was done by EAA, RER, and MB. RER, EAA, and MB drafted the review. All authors reviewed and approved the final version of the manuscript.

\section{Conflicts of Interest}

None declared.

\section{Multimedia Appendix 1}

Search strategies.

[DOCX File, 18 KB-Multimedia Appendix 1]

\section{Multimedia Appendix 2}

Records excluded at the full-text screening phase with full citation.

[DOCX File, $80 \mathrm{~KB}-$ Multimedia Appendix 2]

\section{Multimedia Appendix 3}

Detailed characteristics of included studies.

[DOCX File, 93 KB-Multimedia Appendix 3]

\section{References}


1. Carr C, Hayes R. Social media: defining, developing, and divining. Atl J Commun 2015 Feb 6;23(1):46-65. [doi: 10.1080/15456870.2015.972282]

2. Dol J, Tutelman PR, Chambers CT, Barwick M, Drake EK, Parker JA, et al. Health researchers' use of social media: scoping review. J Med Internet Res 2019 Nov 13;21(11):e13687 [FREE Full text] [doi: 10.2196/13687] [Medline: 31719028]

3. O'Reilly T. What Is Web 2.0: Design Patterns and Business Models for the Next Generation of Software. O'Reilly Media Inc. 2005. URL: http://www.oreilly.com/pub/a/web2/archive/what-is-web-20.html [accessed 2016-11-28]

4. Kuruvilla S, Mays N, Pleasant A, Walt G. Describing the impact of health research: a research impact framework. BMC Health Serv Res 2006 Oct 18;6:134 [FREE Full text] [doi: 10.1186/1472-6963-6-134] [Medline: 17049092]

5. Davidson PM, Newton PJ, Ferguson C, Daly J, Elliott D, Homer C, et al. Rating and ranking the role of bibliometrics and webometrics in nursing and midwifery. ScientificWorldJournal 2014;2014:135812 [FREE Full text] [doi:

10.1155/2014/135812] [Medline: 24550691]

6. Rivera SC, Kyte DG, Aiyegbusi OL, Keeley TJ, Calvert MJ. Assessing the impact of healthcare research: a systematic review of methodological frameworks. PLoS Med 2017 Aug;14(8):e1002370 [FREE Full text] [doi: 10.1371/journal.pmed.1002370] [Medline: 28792957]

7. Scarlat MM, Mavrogenis AF, Pećina M, Niculescu M. Impact and alternative metrics for medical publishing: our experience with international orthopaedics. Int Orthop 2015 Aug;39(8):1459-1464. [doi: 10.1007/s00264-015-2766-y] [Medline: 25947897]

8. Gruzd A, Staves K, Wilk A. Tenure and promotion in the age of online social media. Proc Am Soc Info Sci Tech 2012 Jan 11;48(1):1-9 [FREE Full text] [doi: 10.1002/meet.2011.14504801154]

9. Carrigan M. Social Media for Academics. First Edition. Thousand Oaks, CA: Sage Publications; 2016.

10. Constantinides E, Fountain SJ. Web 2.0: conceptual foundations and marketing issues. J Direct Data Digit Mark Pract 2008 Jan 4;9(3):231-244. [doi: 10.1057/palgrave.dddmp.4350098]

11. Miah A. The A to Z of Social Media for Academia. Academic \& University News: Times Higher Education. 2017. URL: https://www.timeshighereducation.com/a-Z-social-media [accessed 2019-07-03]

12. Champieux R. PlumX. J Med Libr Assoc 2015 Jan;103(1):63-64 [FREE Full text] [doi: 10.3163/1536-5050.103.1.019]

13. Thelwall M. A history of webometrics. Bul Am Soc Info Sci Tech 2012 Aug 21;38(6):18-23. [doi: 10.1002/bult.2012.1720380606]

14. Priem J, Taraborelli D, Groth P, Neylon C. Altmetrics: A Manifesto. Altmetrics. 2011. URL: http://altmetrics.org/manifesto [accessed 2020-05-25]

15. Brigham TJ. An introduction to altmetrics. Med Ref Serv Q 2014;33(4):438-447. [doi: 10.1080/02763869.2014.957093] [Medline: 25316077]

16. Haustein S, Peters I, Sugimoto CR, Thelwall M, Larivière V. Tweeting biomedicine: an analysis of tweets and citations in the biomedical literature. J Assn Inf Sci Tec 2013 Nov 26;65(4):656-669. [doi: 10.1002/asi.23101]

17. Eysenbach G. Can tweets predict citations? Metrics of social impact based on Twitter and correlation with traditional metrics of scientific impact. J Med Internet Res 2011 Dec 19;13(4):e123 [FREE Full text] [doi: 10.2196/jmir.2012] [Medline: $22173204]$

18. Niyazov Y, Vogel C, Price R, Lund B, Judd D, Akil A, et al. Open access meets discoverability: citations to articles posted to academia.edu. PLoS One 2016;11(2):e0148257 [FREE Full text] [doi: 10.1371/journal.pone.0148257] [Medline: 26886730]

19. Kudlow P, Dziadyk DB, Rutledge A, Shachak A, Eysenbach G. The citation advantage of promoted articles in a cross - publisher distribution platform: a 12 - month randomized controlled trial. J Assoc Inf Sci Technol 2019 Dec 23;1:18. [doi: $10.1002 /$ asi.24330]

20. Misirlis N, Vlachopoulou M. Social media metrics and analytics in marketing - S3M: a mapping literature review. Int J Inf Manag 2018 Feb;38(1):270-276. [doi: 10.1016/j.ijinfomgt.2017.10.005]

21. Sugimoto CR, Work S, Larivière V, Haustein S. Scholarly use of social media and altmetrics: a review of the literature. J Assoc Inf Sci Technol 2017 Jun 22;68(9):2037-2062. [doi: 10.1002/asi.23833]

22. Karanatsiou D, Misirlis N, Vlachopoulou M. Bibliometrics and altmetrics literature review. Perform Meas Metr 2017 Apr 10;18(1):16-27. [doi: 10.1108/PMM-08-2016-0036]

23. El Rassi R, Elie A, Bardus M, Meho L, Farha A. The Use of Social Media for Disseminating Health Research. University of York. 2017 Feb 20. URL: http://www.crd.york.ac.uk/PROSPERO/display record.php?ID=CRD42017057709 [accessed 2020-05-25]

24. Patthi B, Prasad M, Gupta R, Singla A, Kumar JK, Dhama K, et al. Altmetrics - a collated adjunct beyond citations for scholarly impact: a systematic review. J Clin Diagn Res 2017 Jun;11(6):ZE16-ZE20 [FREE Full text] [doi: 10.7860/JCDR/2017/26153.10078] [Medline: 28764311]

25. Ruano J, Aguilar-Luque M, Gómez-Garcia F, Mellado PA, Gay-Mimbrera J, Carmona-Fernandez PJ, et al. The differential impact of scientific quality, bibliometric factors, and social media activity on the influence of systematic reviews and meta-analyses about psoriasis. PLoS One 2018;13(1):e0191124 [FREE Full text] [doi: 10.1371/journal.pone.0191124] [Medline: 29377889] 
26. Nass S, Levit L, Gostin L. The value, importance, and oversight of health research. In: Beyond the HIPAA Privacy Rule: Enhancing Privacy, Improving Health Through Research. Washington, DC: The National Academies Press; 2009.

27. Kaplan AM, Haenlein M. Users of the world, unite! The challenges and opportunities of social media. Bus Horiz 2010 Jan;53(1):59-68. [doi: 10.1016/j.bushor.2009.09.003]

28. Bardus M. The web 2.0 and social media technologies for pervasive health communication: are they effective? Stud Commun Sci 2011;11(1):136 [FREE Full text] [doi: 10.5169/seals-791189]

29. Gwet K. Inter-rater reliability: dependency on trait prevalence and marginal homogeneity. Stat Methods Inter-Rater Reliab Assess Ser 2002 May;2:1-9. [doi: 10.1007/springerreference 183942]

30. Gwet K. Handbook of Inter-Rater Reliability: The Definitive Guide to Measuring the Extent of Agreement Among Raters. Simcoe St, Toronto: Advanced Analytics; 2014.

31. Wilson DB. Practical Meta-Analysis Effect Size Calculator. The Campbell Collaboration. URL: http://www. campbellcollaboration.org/escalc/html/EffectSizeCalculator-SMD5.php [accessed 2019-07-06]

32. Amath A, Ambacher K, Leddy JJ, Wood TJ, Ramnanan CJ. Comparing alternative and traditional dissemination metrics in medical education. Med Educ 2017 Sep;51(9):935-941. [doi: 10.1111/medu.13359] [Medline: 28719136]

33. Ramnanan CJ, Ambacher K, Amanth A, O'Brien C, Wood TJ, Leddy JJ. How do alternative and traditional dissemination metrics compare in medical education scholarship? FASEB J 2016;30(1_supplement):568.1 [FREE Full text] [doi:

10.1096/fasebj.30.1 supplement.568.1]

34. Hayon S, Tripathi H, Stormont IM, Dunne MM, Naslund MJ, Siddiqui MM. Twitter mentions and academic citations in the urologic literature. Urology 2019 Jan;123:28-33. [doi: 10.1016/j.urology.2018.08.041] [Medline: 30278190]

35. Knight S. Social media and online attention as an early measure of the impact of research in solid organ transplantation. Transplantation 2014 Sep 15;98(5):490-496. [doi: 10.1097/TP.0000000000000307] [Medline: 25061904]

36. Knight SR. Social media and online attention as an early measure of the impact of research in solid organ transplantation. Transplantation 2014 Sep 15;98(5):490-496. [doi: 10.1097/TP.0000000000000307] [Medline: 25061904]

37. Nguyen KG, Gross C, Cooperberg M, Katz M, Hittelman A, Syed J, et al. MP69-07 hashtag peer-review: does early social media success correlate with conventional metrics of publication impact? J Urology 2017 Apr;197(4S):e928-e929. [doi: 10.1016/j.juro.2017.02.2303]

38. Nolte AC, Nguyen KA, Perecman A, Katz MS, Kenney PA, Cooperberg MR, et al. Association between twitter reception at a national urology conference and future publication status. Eur Urol Focus 2019 May 15:S2405-S4569. [doi: 10.1016/j.euf.2019.05.004] [Medline: 31103604]

39. O'Connor EM, Nason GJ, O'Kelly F, Manecksha RP, Loeb S. Newsworthiness vs scientific impact: are the most highly cited urology papers the most widely disseminated in the media? BJU Int 2017 Sep;120(3):441-454 [FREE Full text] [doi: 10.1111/bju.13881] [Medline: 28418091]

40. O'Connor EM, Nason GJ, O'Kelly F, Manecksha RP, Loeb S. Newsworthiness vs scientific impact: are the most highly cited urology papers the most widely disseminated in the media? BJU Int 2017 Sep 18;120(3):441-454 [FREE Full text] [doi: 10.1111/bju.13881] [Medline: 28418091]

41. Chen W, Cocskhull F, Bukhari M, Galloway J. If I tweet more about my paper, will I improve my citation index? Rheumatology 2019 Apr 12;58(3):kez107.030. [doi: 10.1093/rheumatology/kez107.030]

42. Chiang AL, Rabinowitz LG, Alakbarli J, Chan WW. Tu1108 social media exposure is independently associated with increased citations of publications in gastroenterology. Gastroenterology 2016 Apr;150(4):S845. [doi: 10.1016/S0016-5085(16)32855-4]

43. Heydarpour P, Shirazi A, Sahraian M. Poster session 2: P888 multiple sclerosis research dissemination in the web: news, blogs, or tweets? Mult Scler 2017 Oct;23(3_suppl):427-679 [FREE Full text] [doi: 10.1177/1352458517731406]

44. Konstantiniuk A, Amrein K. Scientific papers in the social media. Wien Klin Wochenschau 2015 Oct;127(19):815-816 [FREE Full text] [doi: 10.1007/s00508-015-0873-9]

45. Matava C, Salman M. The impact of social media on pediatric anesthesia papers and bibliometry. Anesth Analg 2017 May;124(1):S152 [FREE Full text]

46. Fox CS, Bonaca MA, Ryan JJ, Massaro JM, Barry K, Loscalzo J. A randomized trial of social media from circulation. Circulation 2015 Jan 6;131(1):28-33. [doi: 10.1161/CIRCULATIONAHA.114.013509] [Medline: 25406308]

47. Fox CS, Gurary EB, Ryan J, Bonaca M, Barry K, Loscalzo J, et al. Randomized controlled trial of social media: effect of increased intensity of the intervention. J Am Heart Assoc 2016 Apr 27;5(5):- [FREE Full text] [doi:

10.1161/JAHA.115.003088] [Medline: 27121850]

48. Allen HG, Stanton TR, di Pietro F, Moseley GL. Social media release increases dissemination of original articles in the clinical pain sciences. PLoS One 2013;8(7):e68914 [FREE Full text] [doi: 10.1371/journal.pone.0068914] [Medline: $\underline{23874810}$ ]

49. Tonia T, van Oyen H, Berger A, Schindler C, Künzli N. If I tweet will you cite? The effect of social media exposure of articles on downloads and citations. Int J Public Health 2016 May;61(4):513-520. [doi: 10.1007/s00038-016-0831-y] [Medline: 27193574] 
50. Cawcutt KA, Erdahl LM, Englander MJ, Radford DM, Oxentenko AS, Girgis L, et al. Use of a coordinated social media strategy to improve dissemination of research and collect solutions related to workforce gender equity. J Womens Health (Larchmt) 2019 Jun;28(6):849-862. [doi: 10.1089/jwh.2018.7515] [Medline: 30998087]

51. Thoma B, Murray H, Huang SY, Milne WK, Martin LJ, Bond CM, et al. The impact of social media promotion with infographics and podcasts on research dissemination and readership. CJEM 2018 Mar;20(2):300-306. [doi:

10.1017/cem.2017.394] [Medline: 28899440]

52. Hoang JK, McCall J, Dixon AF, Fitzgerald RT, Gaillard F. Using social media to share your radiology research: how effective is a blog post? J Am Coll Radiol 2015 Jul;12(7):760-765. [doi: 10.1016/j.jacr.2015.03.048] [Medline: 25959491]

53. Araújo R, Sorensen AA, Konkiel S, Bloem BR. Top altmetric scores in the Parkinson's disease literature. J Parkinsons Dis 2017;7(1):81-87 [FREE Full text] [doi: 10.3233/JPD-179000] [Medline: 28222540]

54. Araujo AC, Nascimento DP, Gonzalez GZ, Maher CG, Costa LO. Impact of low back pain clinical trials measured by the altmetric score: cross-sectional study. J Med Internet Res 2018 Apr 5;20(4):e86 [FREE Full text] [doi: 10.2196/jmir.9368] [Medline: 29622526]

55. Azer SA, Azer S. Top-cited articles in medical professionalism: a bibliometric analysis versus altmetric scores. BMJ Open 2019 Jul 31;9(7):e029433 [FREE Full text] [doi: 10.1136/bmjopen-2019-029433] [Medline: 31371297]

56. Baan CC, Dor FJ. The transplantation journal on social media: the transplantjrnl journey from impact factor to Klout score. Transplantation 2017 Jan;101(1):8-10. [doi: 10.1097/TP.0000000000001581] [Medline: 27906823]

57. Batooli Z, Ravandi SN, Bidgoli MS. Evaluation of scientific outputs of Kashan university of medical sciences in Scopus citation database based on Scopus, ResearchGate, and Mendeley scientometric measures. Electron Physician 2016 Feb;8(2):2048-2056 [FREE Full text] [doi: 10.19082/2048] [Medline: 27054017]

58. Calopedos RJ, Garcia C, Rashid P, Murphy DG, Lawrentschuk N, Woo HH. Citation indices for social media articles in urology. BJU Int 2017 May;119(Suppl 5):47-52 [FREE Full text] [doi: 10.1111/bju.13872] [Medline: 28544295]

59. Chang J, Desai N, Gosain A. Correlation between altmetric score and citations in pediatric surgery core journals. J Surg Res 2019 Nov;243:52-58. [doi: 10.1016/j.jss.2019.05.010] [Medline: 31154133]

60. Cho J. A comparative study of the impact of Korean research articles in four academic fields using altmetrics. Perform Meas Metr 2017 Apr 10;18(1):38-51. [doi: 10.1108/PMM-02-2016-0005]

61. Costas R, Zahedi Z, Wouters P. Do 'altmetrics correlate with citations? Extensive comparison of altmetric indicators with citations from a multidisciplinary perspective. J Assn Inf Sci Tec 2014 Jul 28;66(10):2003-2019. [doi: 10.1002/asi.23309]

62. Dal-Ré R, Mahillo-Fernández I. Are article's social media mentions associated to citation counts? An analysis in highly influential medical journals. Rev Clin Esp 2018;218(1):40-42. [doi: 10.1016/j.rce.2017.04.003] [Medline: 28571841]

63. Dardas LA, Woodward A, Scott J, Xu H, Sawair FA. Measuring the social impact of nursing research: an insight into altmetrics. J Adv Nurs 2019 Jul;75(7):1394-1405. [doi: 10.1111/jan.13921] [Medline: 30507052]

64. Delli K, Livas C, Spijkervet F, Vissink A. Measuring the social impact of dental research: an insight into the most influential articles on the web. Oral Dis 2017 Nov;23(8):1155-1161. [doi: 10.1111/odi.12714] [Medline: 28734099]

65. Hassona Y, Qutachi T, Dardas L, Alrashdan MS, Sawair F. The online attention to oral cancer research: an altmetric analysis. Oral Dis 2019 Sep;25(6):1502-1510. [doi: 10.1111/odi.13111] [Medline: $\underline{31033085]}$

66. Haustein S, Costas R, Larivière V. Characterizing social media metrics of scholarly papers: the effect of document properties and collaboration patterns. PLoS One 2015;10(3):e0120495 [FREE Full text] [doi: 10.1371/journal.pone.0120495] [Medline: 25780916]

67. Jabaley C, Groff RT, Stentz M, Moll V, Lynde G, Blum J, et al. Highly visible sepsis publications from 2012 to 2017 : analysis and comparison of altmetrics and bibliometrics. J Crit Care 2018 Dec;48:357-371. [doi: 10.1016/j.jcrc.2018.09.033] [Medline: $\underline{30296750]}$

68. Jadhav AP, Ducruet AF, de Leacy R, Fargen KM. Social media and predictors of traditional citations: insights from the journal of neurointerventional surgery. J Neurointerv Surg 2019 Feb;11(2):99-100. [doi: 10.1136/neurintsurg-2018-014689] [Medline: 30665984$]$

69. Jeong JW, Kim MJ, Oh H, Jeong S, Kim MH, Cho JR, et al. The impact of social media on citation rates in coloproctology. Colorectal Dis 2019 Oct;21(10):1175-1182. [doi: 10.1111/codi.14719] [Medline: 31124259]

70. Liu CL, Xu YQ, Wu H, Chen SS, Guo JJ. Correlation and interaction visualization of altmetric indicators extracted from scholarly social network activities: dimensions and structure. J Med Internet Res 2013 Nov 25;15(11):e259 [FREE Full text] [doi: 10.2196/jmir.2707] [Medline: 24275693]

71. Livas C, Delli K. Looking beyond traditional metrics in orthodontics: an altmetric study on the most discussed articles on the web. Eur J Orthod 2018 Apr 6;40(2):193-199. [doi: 10.1093/ejo/cjx050] [Medline: 29016742]

72. Maggio LA, Leroux TC, Meyer HS, Artino AR. \#MedEd: exploring the relationship between altmetrics and traditional measures of dissemination in health professions education. Perspect Med Educ 2018 Aug;7(4):239-247 [FREE Full text] [doi: 10.1007/s40037-018-0438-5] [Medline: 29949099]

73. Punia V, Aggarwal V, Honomichl R, Rayi A. Comparison of attention for neurological research on social media vs academia: an altmetric score analysis. JAMA Neurol 2019 Jul 1:1122-1124 epub ahead of print. [doi: 10.1001/jamaneurol.2019.1791] [Medline: $\underline{\text { 31260003] }}$ 
74. Quintana DS, Doan NT. Twitter article mentions and citations: an exploratory analysis of publications in the American journal of psychiatry. Am J Psychiatry 2016 Feb 1;173(2):194. [doi: 10.1176/appi.ajp.2015.15101341] [Medline: 26844798]

75. Ramezani-Pakpour-Langeroudi F, Okhovati M, Talebian A. Do highly cited clinicians get more citations when being present at social networking sites? J Educ Health Promot 2018;7:18 [FREE Full text] [doi: 10.4103/jehp.jehp_69_17] [Medline: 29629379]

76. Rosenkrantz AB, Ayoola A, Singh K, Duszak R. Alternative metrics ('altmetrics') for assessing article impact in popular general radiology journals. Acad Radiol 2017 Jul;24(7):891-897. [doi: 10.1016/j.acra.2016.11.019] [Medline: 28256440]

77. Ruan QZ, Chen AD, Cohen JB, Singhal D, Lin SJ, Lee BT. Alternative metrics of scholarly output: the relationship among altmetric score, mendeley reader score, citations, and downloads in plastic and reconstructive surgery. Plast Reconstr Surg 2018 Mar;141(3):801-809. [doi: 10.1097/PRS.0000000000004128] [Medline: 29481413]

78. Scotti V, de Silvestri A, Scudeller L, Abele P, Topuz F, Curti M. Novel bibliometric scores for evaluating research quality and output: a correlation study with established indexes. Int J Biol Markers 2016 Dec 23;31(4):e451-e455. [doi: 10.5301/jbm.5000217] [Medline: 27312588]

79. Shirazi M, Goltaji M. An altmetric study on scientific articles of 'health literacy' in social media. Payesh Health Monit 2018;17(3):249-256 [FREE Full text]

80. Smith ZL, Chiang AL, Bowman D, Wallace MB. Longitudinal relationship between social media activity and article citations in the journal gastrointestinal endoscopy. Gastrointest Endosc 2019 Jul;90(1):77-83. [doi: 10.1016/j.gie.2019.03.028] [Medline: 30935934]

81. Thelwall M, Haustein S, Larivière V, Sugimoto CR. Do altmetrics work? Twitter and ten other social web services. PLoS One 2013;8(5):e64841 [FREE Full text] [doi: 10.1371/journal.pone.0064841] [Medline: 23724101]

82. Thelwall M, Wilson P. Mendeley readership altmetrics for medical articles: an analysis of 45 fields. J Assn Inf Sci Tec 2015 May 5;67(8):1962-1972. [doi: 10.1002/asi.23501]

83. Wiehn J, Southam E. Monitoring the social-media footprint of newly published articles to assess short-term impact. Curr Med Res Opin 2017 May;33(sup1):i-iv. [doi: 10.1080/03007995.2017.1296267]

84. Schober P, Boer C, Schwarte LA. Correlation coefficients: appropriate use and interpretation. Anesth Analg 2018 May;126(5):1763-1768. [doi: 10.1213/ANE.0000000000002864] [Medline: 29481436]

85. Mukaka M. Statistics corner: a guide to appropriate use of correlation coefficient in medical research. Malawi Med J 2012 Sep;24(3):69-71 [FREE Full text] [Medline: 23638278]

86. Evaniew N, Adili AF, Ghert M, Khan M, Madden K, Smith C, et al. The scholarly influence of orthopaedic research according to conventional and alternative metrics: a systematic review. JBJS Rev 2017 May;5(5):e5. [doi: 10.2106/JBJS.RVW.16.00059] [Medline: 28557819]

87. Fenner M. What can article-level metrics do for you? PLoS Biol 2013 Oct;11(10):e1001687 [FREE Full text] [doi: 10.1371/journal.pbio.1001687] [Medline: 24167445]

88. Featherstone R. Scholarly tweets: measuring research impact via altmetrics. J Can Health Libr Assoc 2014 Aug 1;35(2):60. [doi: $10.5596 / \mathrm{c} 14-015]$

\section{Abbreviations}

AAS: Altmetrics attention score

DOI: digital object identifier

IF: impact factor

PROSPERO: International Prospective Register of Systematic Reviews

RCT: randomized controlled trial

RIF: research impact framework

Edited by G Eysenbach; submitted 24.07.19; peer-reviewed by EM Messner, A Barke, J McCall, A Ketchum, E Yan; comments to
author $28.10 .19 ;$ revised version received 30.12.19; accepted 29.03.20; published 06.07.20
Please cite as:
Bardus $M$, El Rassi R, Chahrour M, Akl EW, Raslan AS, Meho LI, Akl EA
The Use of Social Media to Increase the Impact of Health Research: Systematic Review
J Med Internet Res 2020;22(7):e15607
URL: $\underline{\text { https://www.jmir.org/2020/7/e15607 }}$
doi: $\underline{10.2196 / 15607}$
PMID: $\underline{32628113}$


CMarco Bardus, Rola El Rassi, Mohamad Chahrour, Elie W Akl, Abdul Sattar Raslan, Lokman I Meho, Elie A Akl. Originally published in the Journal of Medical Internet Research (http://www.jmir.org), 06.07.2020. This is an open-access article distributed under the terms of the Creative Commons Attribution License (https://creativecommons.org/licenses/by/4.0/), which permits unrestricted use, distribution, and reproduction in any medium, provided the original work, first published in the Journal of Medical Internet Research, is properly cited. The complete bibliographic information, a link to the original publication on http://www.jmir.org/, as well as this copyright and license information must be included. 